\title{
Optical Light Curves of the Type Ia Supernovae 1990N and 1991T
}

\author{
P. Lira ${ }^{1}$, Nicholas B. Suntzeff, M. M. Phillips, Mario Hamuy ${ }^{2}$ \\ Electronic mail: p.lira@roe.ac.uk, nsuntzeff@noao.edu, mphillips@noao.edu, \\ mhamuy@as.arizona.edu \\ Cerro Tololo Inter-American Observatory, National Optical Astronomy Observatories, ${ }^{3}$ \\ Casilla 603, La Serena, Chile \\ and \\ José Maza \\ Dept. Astronomía, Universidad de Chile, Casilla 36-D, Santiago, Chile \\ Electronic mail: jmaza@das.uchile.cl \\ and \\ R. A. Schommer, R. C. Smith ${ }^{4}$, Lisa A. Wells ${ }^{3}$, \\ R. Avilés, J. A. Baldwin, J. H. Elias ${ }^{5}$, L. González, A. Layden ${ }^{4,6}$, M. Navarrete, P. Ugarte, \\ Alistair R. Walker, Gerard M. Williger ${ }^{7}$ \\ Cerro Tololo Inter-American Observatory, National Optical Astronomy Observatories, ${ }^{2}$ \\ Casilla 603, La Serena, Chile \\ and \\ F. K. Baganoff \\ Center for Space Research 37-518A, Massachusetts Institute of Technology, 77 Massachusetts \\ Avenue, Cambridge, MA 02139 \\ and \\ Arlin P. S. Crotts, R. Michael Rich, N. D. Tyson ${ }^{8}$ \\ Department of Astronomy, Columbia University, Mail Code 5242, New York, NY 10027 \\ and \\ A. Dey
}

Kitt Peak National Observatory, National Optical Astronomy Observatories, 950 N. Cherry Ave, Tucson, Arizona 85726

and

P. Guhathakurta

UCO/Lick Observatory, University of California, 1156 High Street, Santa Cruz, California 95064

and 


\section{J. Hibbard}

Institute for Astronomy, University of Hawai'i, 2680 Woodlawn Drive, Honolulu, Hawai'i 96822

and

Y. -C. Kim

Astronomy Department, Yale University, P. O. Box 208101, New Haven, Connecticut 06520

and

Daniel M. Rehner, E. Siciliano

Space Telescope Science Institute, 3700 San Martin Drive, Baltimore, Maryland 21218

and

Joshua Roth

Sky Publishing Corporation, 49 Bay State Road, Cambridge, MA 02138

and

Patrick Seitzer

Dept. of Astronomy, University of Michigan, 830 Dennison Bldg., Ann Arbor, Michigan 48109

and

T. B. Williams

Physics and Astronomy Dept., Rutgers University, P. O. Box 849, Piscataway, New Jersey 08855

\footnotetext{
${ }^{1}$ Current address: Institute for Astronomy, University of Edinburgh, Royal Observatory, Blackford Hill, Edinburgh EH9 3HJ, UK

${ }^{2}$ Current address: University of Arizona, Steward Observatory, Tucson, Arizona, 85721

3 Cerro Tololo Inter-American Observatory, National Optical Astronomy Observatories, operated by the Association of Universities for Research in Astronomy, Inc., (AURA), under cooperative agreement with the National Science Foundation.

${ }^{4}$ Current address: Department of Astronomy, University of Michigan, Ann Arbor, Michigan 48109

${ }^{5}$ Current address: Kitt Peak National Observatory, National Optical Astronomy Observatories, 950 N. Cherry Ave, Tucson, Arizona 85726

${ }^{6}$ Hubble Fellow

${ }^{7}$ Current Address: National Optical Astronomy Observatories, Code 681, NASA Goddard Space Flight Center, Greenbelt, Maryland 20771 USA

${ }^{8}$ Current address: Princeton University Observatory, Peyton Hall, Princeton, New Jersey 08544

${ }^{9}$ Current address: Harvard-Smithsonian Center for Astrophysics, 60 Garden St. Cambridge, MA 02139
} 


\begin{abstract}
We present UBVRI light curves for the bright Type Ia supernovae SN 1990N in NGC 4639 and SN 1991T in NGC 4527 based on photometry gathered in the course of the Calán/Tololo supernova program. Both objects have well-sampled light curves starting several days before maximum light and spanning well through the exponential tail. These data supercede the preliminary photometry published by Leibundgut $e$ t al. (1991), and Phillips et al. (1992)]. The host galaxies for these supernovae have (or will have) accurate distances based on the Cepheid period-luminosity relationship. The photometric data in this paper provide template curves for the study of general population of Type Ia supernova and accurate photometric indices needed for the Cepheid-supernova distance scale.
\end{abstract}

\title{
1. Introduction
}

It is now clear that Type Ia supernovae are not a homogenous class of objects. One can see differences in spectral features at specific epochs (Bertola (1964), Branch (1987), Phillips et al. (1987), Harkness \& Wheeler (1990), Nugent et al. (1995), Filippenko (1997)] and in the overall morphology of the light curves (Phillips et al. (1987), Phillips (1993), Suntzeff (1995)]), which had long been suspected by earlier workers (Barbon et al. 1973, Rust (1974), Pskovskii (1977), Branch (1981), Pskovskii (1984)]. The modern data have shown us, however, that the class of Type Ia supernovae can still be used to provide accurate relative distances by applying correction factors to the observed luminosity which are a simple function of the evolution of the light curve near maximum light (Phillips (1993), Hamuy et al. (1995), Riess et al. (1995)). The simple proof that these techniques work is the reduction in the magnitude scatter of a Hubble diagram for distant supernovae, where the scatter reduces from about $0.4 \mathrm{mag}$ to $0.14 \mathrm{mag}$ (Hamuy et al. (1996b), Riess et al. (1996)).

To use the very high accuracy of the zero-point of the distant supernova Hubble diagram to measure an accurate absolute distance scale requires the direct measurements of the distances to a number of nearby galaxies which have had well-observed Type Ia supernovae. One of the most accurate methods to measure absolute distances to nearby galaxies is the use of the Cepheid period-magnitude relationship calibrated relative to the Large Magellanic Cloud (Madore \& Freedman (1991)]. With an independent measurement of the distance to the LMC the observed Hubble diagram of distant supernovae will directly yield the Hubble constant. The nearby calibrating galaxies must have three rather trivial properties: the galaxy must be young enough to form classical Cepheids; the galaxy must have hosted a reasonably "normal" type Ia supernova; and the supernova light curve must have been reasonably well-measured.

There are only a handful of such supernova host galaxies within the light grasp of HST, where the limiting distance modulus for measuring Cepheids light curves is about 32.0. Six supernovae, 
SNe 1895B, 1937C, 1960F, 1972E, 1981B, and 1990N have been calibrated to date by Saha, Sandage and collaborators (see Saha et al. (1996) for the most recent paper in this series).

We have pointed out (Hamuy et al. (1996b) that a few of the nearby supernovae are not ideal as calibrators. The light curves for SNe 1895B and 1960F are very poor with ill-defined maxima. SN 1937C was well observed, but the transformation from the 60 year-old films to modern photometric bandpasses, while carefully calibrated (Pierce \& Jacoby (1995)]), remains controversial in some circles (Schaefer (1996); rebuttal in Jacoby \& Pierce (1996)). Both 1937C and 1972E clearly had "slower" evolution near maximum light, which we have shown is indicative of intrinsically brighter supernovae (Hamuy et al. (1996a)); however, this latter point has been contested (Tammann \& Sandage (1995), Sandage et al. (1996)]. SN 1989B had very high reddening $(E(B-V) \sim 0.4$; Wells et al. 1994). Only SNe 1981B and 1990N have evidently both uncontroversial light curves and Cepheid distances.

If we relax the requirement that the Cepheids must be measured in the same galaxy as the supernova and rely on group or cluster associations between galaxies, a number of other calibrators become available. Cepheid distances to NGC 3368 (M96) (Tanvir et al. (1995) and NGC 3351 (Graham et al. (1997) have been measured with HST data. Sandage et al. (1996) associate the M96 group with a larger Leo Group (also called the Leo I cloud) which includes the compact M66 (Leo Triplet) group. However, M66, which hosted SN 1989B, is some $8^{\circ}$ away from the Cepheid host galaxies and one must worry that the whole Leo group is at the same distance. HST observations to determine a Cepheid distance to M66 are planned for HST Cycle 7 by Saha, Sandage, and collaborators. There have also been two well studied Type Ia supernovae in the Fornax cluster: SN 1980N (in NGC 1316) and SN 1992A (in NGC 1380). Silbermann et al. (1996) have measured a Cepheid distance to the peculiar spiral NGC 1365 thought to be a member of Fornax. Once again, the physical association of the supernova host galaxy and the Cepheid host galaxy is a point of some controversy. Such ambiguities lead us to prefer Type Ia supernova absolute magnitude calibrations based on galaxies which have both primary calibrators such as Cepheids and supernova.

SN 1990N was discovered significantly before maximum in the SBb(r) galaxy NGC 4639 by E. Thouvenot at the Observatory of the Côte d'Azur on 22 June 1990 (Maury (1990); all dates referenced as UT). Pollas (1990) measured an astrometric position for this supernova of $(\mathrm{RA}$, dec,equinox $)=\left(15^{h} 18^{m} 52^{\mathrm{s}} .92,-7^{\circ} 11^{\prime} 43^{\prime \prime} .2,1950\right)$. Kirshner \& Leibundgut (1990) classified it as a Type Ia supernova on the basis of a spectrum obtained on the 26 June 1990. Leibundgut $e$ al. (1991) presented preliminary light curves based on CTIO CCD data (which is reanalyzed in the present paper). Spectral modeling has been published by Jeffery et al. (1992), Shigeyama et al. (1992), Yamaoka et al. (1992), and Fisher et al. (1997). Recently, Sandage et al. (1996) and Saha et al. (1996) have obtained the distance to the parent galaxy by measuring the periods and magnitudes of 20 Cepheid variable stars with the Hubble Space Telescope. This object is therefore a key template objects in establishing the value of $H_{0}$ based on the Cepheid-supernova distance scale. 
SN $1991 \mathrm{~T}$ has been one of the most extensively studied Type Ia supernovae. It was discovered well before maximum in the $\mathrm{Sb}(\mathrm{s}) \mathrm{II}$ galaxy NGC 4527 by S. Knight and independent observers (Waagen et al. (1991) on 13 April 1991. An astrometric position for this supernova by R. H. McNaught is given in the previous reference as $(\mathrm{RA}$,dec,equinox $)=\left(12^{h} 31^{m} 36^{\mathrm{s}} .91,+2^{\circ} 56^{\prime} 28^{\prime \prime} \cdot 3\right.$, 2000).

Early optical spectral observations reported by La Franca \& Goldschmidt (1991), Kirshner (1991), and Phillips \& Hamuy (1991) showed that SN 1991T was a peculiar Type Ia event which motivated a number of theoretical studies to model the spectral evolution (Jeffery et al. (1992), Ruiz-Lapuente et al (1992), Spyromilio et al. (1992), Yamaoka et al. (1992), Mazzali et al. (1995), Meikle et al. (1996)]). Optical photometry has been published by Phillips et al. (1992), Ford et al. (1993), and Schmidt et al. (1994) which showed that SN 1991T had a very slow rate of evolution through maximum. Due to the excellent temporal coverage of the light curve, this supernova has been used as template example of a slow supernova (Hamuy et al. (1996c), Riess et al. (1996)). It is expected that the Saha and Sandage group will obtain a Cepheid distance to NGC 4527 using data taken with HST in Cycle 7.

When accurate modern light curves for several nearby supernovae became available some years ago, subtle differences between Type Ia supernova light curves became apparent. CCD photometry showed that there is a real spread in the peak luminosity and that some of the objects evolve through maximum light more slowly than others. Phillips (1993) presented evidence that the rate of the decline after maximum is correlated with the luminosity at maximum and that more luminous objects have a slower decline rates. Hamuy et al. (1995), Riess et al. (1995), Hamuy et al. (1996b), and Riess et al. (1996) have found that the scatter in the Hubble diagram of Type Ia supernovae decreases significantly when corrections for the peak luminosity - decline rate relation are introduced. Tammann \& Sandage (1995] argue that when samples are restricted to "normal" objects (by eliminating events like SN 1991T) there is no need to correct for a peak luminosity - decline rate effect. However, the Hamuy studies find that by ignoring this effect, the estimate of the Hubble constant can be biased too low by up to $15 \%$.

The intent of this paper is to present accurate light curves of the two nearby supernovae SNe $1990 \mathrm{~N}$ and $1991 \mathrm{~T}$ which are important calibrators in the distance scale. We have already used these light curves in our work on the Hubble constant (Hamuy et al. (1996b). In Section 2 of this paper we present the observations and reduction of the optical photometric data obtained at CTIO. The light curves as well as color curves for both supernovae are shown in Section 3. A final discussion is found in Section 4.

\section{Observations}

The optical observations of SNe $1990 \mathrm{~N}$ and $1991 \mathrm{~T}$ were obtained using the $0.9 \mathrm{~m}$ and the Blanco 4m telescopes at CTIO. SN 1990N was observed from June 1990 to March 1992 and SN 
$1991 \mathrm{~T}$ was observed from April 1991 to June 1992. The observations were made using Texas Instrument and Tektronix CCDs (except for the night of the 19 March 1990 when a Thomson detector was used) and facility $U B V(R I)_{K C}$ filters in the Johnson-Kron-Cousin photometric system (Johnson (1963), Kron (1953), Cousins (1976). The observation logs are given in Table 11 and 2. The detector name, listed in the final column of these two tables, combines the manufacturer name and a running number assigned by the CTIO CCD lab. We have assumed that each different CCD listed in this table (along with the filter set) has a unique set of color terms that must be derived from observations.

We made observations of the supernovae under varied photometric conditions, including very non-photometric weather with cloud extinction up to a few magnitudes. It is well established (Serkowski (1970), Walker et al. (1970), Olsen (1983)]) that clouds are quite grey, which allows us to use local standards (in the same CCD frame as the supernova) and averaged color terms for a specific CCD measured on photometric nights.

For accurate photometry on non-photometric nights, it is necessary to define a precise local photometric sequence of stars. We measured photometric sequences on 13 photometric nights in the CCD field around NGC 4639 and NGC 4527 referenced to the Landolt and Graham standards stars (Landolt (1972), Graham (1982), Landolt (1992)). Extinction coefficients, color terms and zero points for the transformations to the standard $U B V(R I)_{K C}$ system were derived for each night following the method described by Harris et al. (1981). Typical values of the extinction coefficients were $k_{U}=0.50, k_{B}=0.32, k_{V}=0.20, k_{R}=0.14$ and $k_{I}=0.08$ in units of mag (airmass) $^{-1}$. 四 We measured $U B V(R I)_{K C}$ sequences for a total of 15 stars for $\mathrm{SN} 1990 \mathrm{~N}$ and 9 stars for SN $1991 \mathrm{~T}$ using digital aperture photometry with an aperture diameter of $14^{\prime \prime}$. The photometric sequences are identified in Figures 1 and 2, and the photometry is given in Tables 3 and 1 . In these tables we list the number of observing nights (n) and the total number of observations $(\mathrm{m})$.

Star 2 in our local sequence around SN $1991 \mathrm{~T}$ is also a sequence star (number 2) listed by Ford et al. (1993). The magnitude differences for this star in the sense of this study minus Ford et al. $(1993)$ are $\Delta(V R I)=(0.00,-0.02,-0.03)$. For the three SN $1991 \mathrm{~T}$ local standards in common with Schmidt et al. (1994), we find the mean differences in the sense of this study minus that of Schmidt et al. (1994) are $\Delta(B V R I)=(-0.03 \pm 0.01,-0.04 \pm 0.01,-0.02 \pm 0.02,+0.12 \pm 0.08)$ where the errors quoted are the mean errors. These mean differences are consistent with the photometric errors quoted in Schmidt et al. (1994), which dominate the comparison of the two magnitude systems. Due to the larger number of measurements on independent photometric nights, we are confident that sequences given in Tables 3 and 6 are the most accurate available.

To determine the supernova magnitudes we subtracted late-time images of the parent galaxies

\footnotetext{
${ }^{10}$ These extinction values are higher than normal due to the effects of the Mt. Pinatubo eruption which occurred on JD 2448422. See Grothues \& Gochermann (1992).
} 
at the location of the supernovae using the technique described by Hamuy et al. (1994). For these subtractions deep "master images" of NGC 4639 and NGC 4527 were obtained at the beginning of 1994, which corresponds to 1300 and 1010 days after maximum light for SNe 1990N and 1991T. A simple extrapolation of the late-time decline rates (see the $\gamma$ parameter in Table 8) to these dates yields $B$ magnitudes of $\sim 33(\mathrm{SN} 1990 \mathrm{~N})$ and $\sim 28(\mathrm{SN} 1991 \mathrm{~T})$. However, there are two factors which could make the late-time magnitudes in the master images significantly brighter than this extrapolation

The first factor is the presence of minor radioactive nuclides and the efficiency of positron energy deposition from the radioactive decays. A Type Ia explosion is predicted to synthesize about $0.5 \mathrm{M} \odot$ of ${ }^{56} \mathrm{Ni}$, and smaller amounts of ${ }^{56} \mathrm{Co},{ }^{57} \mathrm{Co},{ }^{44} \mathrm{Ti}$, and ${ }^{22} \mathrm{Na}$ (see model W7 in Nomoto et al. (1994)). Woosley et al. 1989 provide energy deposition rates for these nuclides. A complication arises in predicting the late-time light curve after day 500 in how to handle the energy deposition from the positron production (Arnett (1979). The positrons can add energy into the supernova nebula both from their kinetic energies and annihilations. The efficiency of this process is poorly understood in the low-density environment of the supernova nebula at late-time. If we make the rather extreme assumption of complete kinetic energy deposition and positron annihilation into gamma rays, we can use the deposition rates given by Woosley et al. 1989 and the model W7 abundances by Nomoto et al. (1994) to predict upper limits to the supernova luminosity at late time. The effect of full energy deposition from positrons and the existence of long-lived radioactive nuclides such as ${ }^{44} \mathrm{Ti}$, and ${ }^{22} \mathrm{Na}$ tends to flatten out the light curve past day 1000. Using a column depth of $400 \mathrm{~g} \mathrm{~cm}^{-2}$ at $\mathrm{t}=10^{6} \mathrm{~s}$ as suggested by Woosley et al. 1989 for a Type Ia supernova, we predict $V$ magnitudes of 29.6 and 25.4 for SNe 1990N and 1991T at the epoch of the master image. For no positron energy deposition, the predicted magnitudes are about 3 magnitudes fainter. In either case, the predicted magnitudes in the master images are so faint as to have no effect on the measured photometry. However, if there is significant overproduction of ${ }^{57} \mathrm{Co}$ or ${ }^{44} \mathrm{Ti}$ relative to model $\mathrm{W} 7$, the late-time magnitudes could be much brighter and affect the magnitudes measured by image subtraction.

The second factor that could affect the magnitudes measured from the subtracted images is the presence of a light echo. In the late-time images of SN 1991T, the location of supernova has been found to be contaminated by a faint echo of SN 1991T at maximum light Schmidt et al. (1994)). We will return to this minor complication in Section 3.

We measured differential photometry of the supernovae on each CCD frame using aperture photometry when the supernova was bright, or using the point spread function (psf) fitting program DAOPHOT (Stetson (1987) when the supernova was faint. Averaged color terms chosen to match the CCD/filter setup of instruments for each observing night were adopted from a database of coefficients at CTIO. For SN 1991T near maximum, our CCD exposures were very short and the local standards were poorly exposed. In this case we used the sharp core of NGC 4527 as a $B V$ "standard" for the nights of 26, 28, 29, and 30 April, and 1 May 1991. An aperture radius of $2.7^{\prime \prime}$ was chosen to maximize the signal to noise ratio for the photometry of the core. 
The core photometry is listed in Table 4 .

Besides the error given by the Poisson statistics of the number of counts in the supernova aperture or psf, $\sigma_{\text {phot }}$, there are other error sources such as those due to the transformation of the instrumental magnitudes into the standard system and CCD flat fielding. To get a sense of the magnitude of these errors we selected many frames with a sufficient number of bright stars (with negligible $\sigma_{\text {phot }}$ error). For each frame we calculated the standard deviation of the difference between a given measurement and the standard magnitudes listed in Tables 3 and 4 . This standard deviation $\left(\sigma_{r m s}\right)$ is an empirical estimate of the average error in a single observation of a stellar object in any CCD frame when referenced to the local photometric sequence. The measured standard deviations $\sigma_{r m s}$ were $(0.026,0.017,0.017,0.015,0.017)$ magnitudes in $U B V R I$. The value of $\sigma_{r m s}$ derived for both supernovae in each filter agreed within 0.002 magnitudes).

The final error in the individual magnitudes of the supernovae was calculated as the quadratic sum of the empirical error in a single observation $\sigma_{r m s}$ and the photon statistical error, $\sigma_{p h o t}$. The error $\sigma_{r m s}$ was the dominant component of the errors in the early part of the photometry, while $\sigma_{\text {phot }}$ became more important when the supernova dimmed.

\section{Results}

\section{1. $\quad$ Light Curves}

We present $U B V(R I)_{K C}$ photometry for SNe 1990N and $1991 \mathrm{~T}$ in Tables 5 and 6 , and plot the data in Figures 3 and 4 . To find the time and magnitude of maximum light for both supernovae we fit the data around the peak with a third-order polynomial. For SN 1990N the first observation was acquired 11 days before $B_{\max }$, and the last observation was made 607 days after $B_{\max }$ in just the $V$ band. The $B$ maximum was reached on JD $2448082.7 \pm 0.5$ with $B_{\max }=12.76 \pm 0.03$ and a $B-V$ color of 0.03 magnitudes. For SN 1991T the data begin 12 days before $B_{\max }$ and end 401 days after maximum. We derive $B_{\max }=11.70 \pm 0.02$ at JD $2448375.7 \pm 0.5$ with a $B-V$ color of 0.17. In Table 7 we summarize the maxima of the light curves in the different bands for both supernovae. We find that the $B$ maximum occurs before the $V$ maximum; in particular, the time difference between the $B$ and $V$ maxima is $1.5 \pm 0.7$ days for SN $1990 \mathrm{~N}$ and $2.6 \pm 0.7$ days for SN $1991 \mathrm{~T}$, in agreement with the result of Leibundgut (1988) who found a difference of $2.5 \pm 0.5$ days.

Comparisons of our $B V$ photometry with previous data published for SNe 1990N and 1991T are shown in Figures 5 and 6 . The agreement between the photometry presented in this paper and the results published by Leibundgut et al. (1991) for SN 1990N and Phillips et al. (1992) for SN 1991T are not surprising since they are based on a subsample of the same optical data analyzed here. However, a small systematic difference between the different data sets is clear. Our photometry near maximum is generally dimmer, although the discrepancy is less than 0.1mag for SN 1990N and even less for SN 1991T. The preliminary photometric results of these earlier 
papers which were based on only a single night for the photometric calibration should be ignored in preference to the photometric data given in Tables 5 and 6 .

For SN 1991T there is independent photometry published by Ford et al. (1993) which we plot in Figure 6. If we interpolate our data to the dates of the Ford et al. (1993) data using a spline fit, we find the following differences in the sense of this work minus Ford et al.: $V, 0.08 \pm 0.01$ ; $R, 0.04 \pm 0.02$; and $I, 0.02 \pm 0.02$. The quoted errors are the errors in the mean based on the interpolation to the 12 dates in the Ford et al. study. A similar systematic offset in the $V$ magnitude was noted by Ford et al. with respect to the Phillips et al. (1992) reductions of the $1991 \mathrm{~T}$ data. These mean differences between careful photometric studies indicate the level in systematic errors that can be encountered even in bright supernova photometry.

It is now well established that there is not a unique light curve for all type Ia supernova. As was suggested by Pskovskii (1977) and Branch (1981), supernovae can be discriminated by the rate of decline after maximum. Pskovskii $(1977,1984)$ defined the parameter $\beta$ as the characteristic decline rate during the fast-decline phase of the $B$ supernova light curve, and the parameter $\gamma$ as the rate during the slow-decline phase (see Phillips et al. (1987) for an unambiguous description of these parameters). Phillips (1993) introduced the parameter $\Delta m_{15}$, defined as the decline in magnitude during the 15 days after $B$ maximum. The evidence from nearby supernovae (Phillips (1993), Hamuy et al. (1996c)) and the scatter in the observed Hubble diagram (Maza et al. (1994), Hamuy et al. (1995), Hamuy et al. (1996b) clearly show that the brighter supernova decline more slowly (small $\Delta m_{15}$ ).

In Table 8 we list the evolutionary parameters of the $B$ light curves for our two supernovae. We also list the values for the Leibundgut (1988) template $B$ curve. This multicolor template, which was formed from a large number of supernova light curves, provides a useful fiducial light curve and can be considered a "typical" light curve which can be compared to other observations. We calculated the Pskovskii $\beta$ and $\gamma$ parameters for the $B$ curves of SNe 1990N and 1991T using a linear least-squares fit with the data weighted using the photometric errors as quoted in Tables 5 and 6. The range of days used in the fitting for each supernova are indicated in Table 8 .

Table 8 shows that in both the $\beta(B)$ and $\Delta m_{15}$ are much smaller for SN $1991 \mathrm{~T}$ than the values for the Leibundgut template. Phillips (1993) has shown that this "slow" supernova was intrinsically very bright. In fact, SN 1991T is one of the slowest supernovae ever found and has been used as a representative template for slow events (Hamuy et al. (1995), Hamuy et al. (1996c)]. SN 1990N, on the other hand, is quite similar to the Leibundgut template, and is therefore similar to the typical Type Ia event.

In Figures 7 and 8 we plot the first 120 days of $B V$ photometry for the two supernovae along with the Leibundgut templates. The $B V$ templates have been shifted to match the epoch of $B$ maxima and the peak magnitudes given in Table of (with the appropriate time delay between $B$ and $V$ maximum given above). The results given in the preceeding paragraph can be now clearly seen in these figures. SN 1990N follows the template closely while SN 1991T declines from 
maximum light more slowly. SN $1991 \mathrm{~T}$ also begins its exponential decline significantly earlier and remains at higher relative brightness when compared to the template. Visually, the "knee" in the light curve around 30 days past maximum occurs earlier in this supernova. SN 1991T also rises to maximum light more slowly than SN 1990N.

In Figure 9 we plot the late-time photometry of SN 1991T from this study and Schmidt et al. (1994) along with the predicted trend of the late-time evolution based on the $\gamma(B)$ and $\gamma(V)$ fits to our data. The fact that the light curve past day 400 levels off has been shown by Schmidt et al. (1994) to be due to a light echo with $B V \sim(21.3,21.4)$. Recall that in our work we subtracted a late-time image of the region taken around JD2449380 around the supernova to remove the galaxian contribution to the background under the psf. By doing this however, we also automatically correct for the echo contamination. This assumption is valid provided that the echo magnitude did not change during the period between the supernova observations and the late-time image, and that the light curve of supernova did not level off for other reasons, such as the overproduction of ${ }^{44} \mathrm{Ti}$ or ${ }^{22} \mathrm{Na}$. Under these assumptions, the photometry of SN $1991 \mathrm{~T}$ in Table 6 and Figure 9 should be free of any echo contamination. Indeed, the small differences between our last points and those of Schmidt et al. at JD 2448750 shown in Figure 9 are consistent with the echo magnitude cited above.

\subsection{Color Curves}

The $B-V, B-R, B-I, V-R, V-I, R-I$ color curves for SNe 1990N and 1991T through day 100 are shown in Figures 10 and 11 respectively. The temporal axis was shifted so that the $B$ maximum corresponds to $t=0$ for both supernovae. The redder color of SN 1991T with respect to SN 1990N is evident. The presence of redshifted Na absorption lines in the spectrum of SN $1991 \mathrm{~T}$ and the location of the supernova in one of the arms of NGC 4527 suggest that this object was obscured by dust in its parent galaxy. Strong Ca and Na interstellar absorption lines at the radial velocity of NGC 4527 were observed by Wheeler \& Smith (1991) and Meyer \& Roth (1991). Ruiz-Lapuente et al (1992) estimated an excess $E(B-V) \sim 0.3$ assuming a relationship between the equivalent width of the line Na I D and $E(B-V)$. On the other hand, Phillips et al. (1992) found an excess of 0.13 magnitudes assuming an intrinsic color $(B-V)$ of zero during maximum. The foreground $E(B-V)$ reddening is $0.00 \pm 0.015$ according to Burstein \& Heiles (1994).

SN 1990N did not show absorption lines in a low-dispersion spectrum (Leibundgut et al. (1991) and its location in the outskirts of NGC 4639 suggests that this object is less reddened than SN 1991T. Saha et al. (1996) estimate the mean extinction of the Cepheids as $E(V-I)=0.04 \pm 0.06$ based on the difference in distance moduli from Cepheid VI P-L relations. The foreground $E(B-V)$ reddening is $0.012 \pm 0.015$ according to Burstein \& Heiles (1994). It is unfortunate that no high-dispersion spectrum of this bright object was made. The $(B-V)$ color of 0.03 is consistent with an intrinsic color at maximum of $\sim-0.1-0.1$ magnitudes for other Type Ia supernovae with low reddenings (Hamuy et al. (1991), Sandage \& Tammann (1993), Hamuy et 
al. (1995)) and suggests $E(B-V) \lesssim 0.15$. Lira (1996) has shown that the color evolution in $B V$ from days 32-92 during the nebular phase is extremely uniform among Type Ia supernovae. In a future paper we will use this fact to calibrate the intrinsic colors of SNe Ia at maximum which, in turn, should allow more precise estimates to be made of the host galaxy reddening.

Differences in the color evolution of the two supernovae are better appreciated in the color-color plot shown in Figure 12. Time along the light curve is indicated by labeling the points at approximately -10, 0, 10 and 20 days from the maximum. The figure also shows the reddening vector for a galactic extinction law (Savage \& Mathis (1979)]). For $t>10$ days, the curves are parallel to the reddening vector. However, the data from $t=-10$ to $t=10$ show that the color curve of SN $1991 \mathrm{~T}$ cannot be matched to that of $1990 \mathrm{~N}$ by a simple dereddening vector.

\section{Concluding Remarks}

SNe 1990N and 1991T are important supernovae. They were close enough that distances to the host galaxies can (or will) be measured by direct techniques with the HST. The light curves are especially well determined over the full evolution, and in particular, the evolution before maximum is well covered. The light curves of these supernovae have become standard templates used in the study of more distant supernovae.

The photometric data presented in this paper show that SN 1990N was a typical Type Ia event in that the light curves are well fitted by the template curve determined by Leibundgut (1988). It also falls in the middle of the range of $\Delta m_{15}$ types defined by Phillips (1993). The spectral evolution of SN 1990N has also been classified as similar to other prototypical Type Ia supernovae, although the early first observations made it to be claimed as a peculiar object (Leibundgut et al. (1991).

The preliminary reductions of the SN 1990N data in Leibundgut et al. (1991) have been used by Sandage et al. (1996) to estimate a peak absolute magnitude for this supernova. The peak magnitudes cited by Sandage et al. of $(B, V)=(12.70,12.61)$ are $\sim 0.07$ mag brighter than the more precise results given in Table 7 . Such a small magnitude difference will have little effect on the measurement of the Hubble constant since $\delta H_{0} / H_{0} \approx 0.46 \delta m$. Hamuy et al. (1996b), Sandage et al. (1996) and Riess et al. (1996) have used the light curve of this supernova as one of the fundamental calibrators of the absolute magnitudes of Type Ia supernovae. These absolute magnitudes coupled with the observed Hubble diagram from the Calán/Tololo survey ( al. (1996b) have yielded $H_{0} \sim 65 \mathrm{~km} \mathrm{~s}^{-1} \mathrm{Mpc}^{-1}$.

Because of its peculiar nature, SN 1991T has been studied intensively. The peculiarities of this supernova include pre-maximum spectra dominated by iron-group features, a very small $\Delta m_{15}$

value, and a visual luminosity larger than other typical Type Ia supernovae, although the derived absolute magnitudes depend strongly on the different extinction assumed for the supernova and the distance to the host galaxy NGC 4527 (Filippenko et al. (1992), Ruiz-Lapuente et al (1992), 
Phillips et al. (1992)]). The results of this paper confirm the slow evolutionary rate near maximum and also show that the color curve is significantly different from more normal Type Ia supernovae.

This is not to say that SN $1991 \mathrm{~T}$ is unique, as new events of this "slow-class" have been found, such as SNe 1991ag (Hamuy et al. (1995)), 1992bc (Maza et al. (1994)), 1995ac (Garvavich et al. (1996) or 1997br Qiao et al. (1997b)). The evidence suggests that the decline rate of these supernovae is just the slow end of the peak luminosity - decline rate relation for Type Ia supernovae and that this correlation could be also extended to a spectroscopic sequence Nugent et al. (1995)]. Hamuy et al. (1996c) and Garvavich et al. (1996) however, have pointed out that the intrinsic luminosity, spectral features, and colors at maximum light are not a simple function of the light curve shape (as measured by $\Delta m_{15}$ ) for this bright class of supernovae. For instance, among supernovae with similar small values of $\Delta m_{15}$, SNe 1991T, 1995ac, and 1997br had very weak Si II 6355 ̊ at maximum light (Filippenko \& Leonard (1995), Qiao et al. (1997a)) while 1992bc had the typical deep spectral features at maximum light common to most Type Ia events (Maza et al. (1994)]). Conversely, SN 1995bd had a spectrum similar to $1991 \mathrm{~T}$ at maximum light but its light curve was well fit by the "faster" Leibundgut template (Garvavich et al. (1996). It is clearly important to obtain more examples of this class of bright Type Ia supernovae to sort out this issue.

JM and MH acknowledge support by Catedra Presidencial de Ciencias 1996-1997. We would like to thank the Space Telescope Science Institute for access to the Digitized Sky Survey. We thank Peter Garnavich, Eric Olsen, Brian Schmidt, and Gordon Walker for helpful correspondence. This research has made extensive use of the Canadian Astronomy Data Center (Dominion Astrophysical Observatory, Herzberg Institute of Astrophysics), and the NASA Astrophysics Data System Abstract Service. We would also like to thank Brian Marsden and Daniel Green at the IAU Central Bureau for Astronomical Telegrams for their valuable notification service which allows observers to start observing supernovae within 24 hours of discovery. 


\section{REFERENCES}

Arnett, W.D. 1979, ApJ, 230, L37

Barbon, R., Ciatti, F., \& Rosino, L. 1973, A\&A, 25, 248

Bertola, F. 1964, Ann. d'Astroph., 27, 319

Branch, D. 1981, ApJ, 248, 1076

Branch, D. 1987, ApJ, 316, L81

Branch, D., Fisher, A., \& Nugent, P. 1993, AJ, 106, 2383

Burstein, D., \& Heiles, C., 1994, ApJS, 54, 33

Cousins, A. W. 1976, MNRAS, 81, 25

Filippenko, A. V., Richmond, M. W., Matheson, T., Shields, J. C., Burbidge, E. M., Cohen, R. D., Dickinson, M., Malkan, M. A., Nelson, B., Pietz, J., Schlegel, D., Schmeer, P., Spinrad, H., Steidel, C. C., Tran, H. D., \& Wren, W. 1992, ApJ, 384, L15

Filippenko, A. V., 1997, ARA\&A, in press.

Filippenko, A.V., \& Leonard, D.C. 1995, IAU Circ.6237

Fisher, A., Branch, D., Nugent, P, \& Baron, E. 1997, ApJ, 481, 89

Ford, C. H., Herbst, W., Richmond, M. W., Baker, M. L., Filippenko, A. V., Treffers, R. R., Paik, Y., \& Benson, P. J. 1993, AJ, 106, 1101

Garnavich, P.M., Riess, A.G., Kirshner, R.P., Challis, P., \& Wagner, R.M. 1996, BAAS, 28, 1331

Grothues, H.-G., \& Gochermann, J. 1992, ESO Messenger, 68, 43

Graham, J. A. 1982, PASP, 94, 244

Graham, J. A., Phelps, R. L., Freedman, W. L., Saha, A., Ferrarese, L., Stetson, P. B., Madore, B. F., Silbermann, N. A., Sakai, S., Kennicutt, R. C., Harding, P., Bresolin, F., Turner, A., Mould, J. R., Rawson, D. M., Ford, H. C., Hoessel, J. G., Han, M., Huchra, J. P., Macri, L. M., Hughes, S. M., Illingworth, G. D., \& Kelson, D. D. 1997, ApJ, 477, 535

Hamuy, M., Phillips, M. M., Maza, J., Wischnjewsky, M., Uomoto, A., Landolt, A. U., \& Khatwani, R. 1991, AJ, 102, 208

Hamuy, M., Phillips, M. M., Maza, J., Suntzeff, N. B., Della Valle, M., Danziger, J., Antezana, R., Wischnjwesky, M., Aviles, R., Schommer, R. A., Kim, Y.-C., Wells, L. A., Ruiz, M. T., Prosser, C. F., Krzeminski, W., Baylin, C. D., Hartigan, P., \& Hughes, J. 1994, AJ, 108, 2226 
Hamuy, M., Phillips, M. M., Maza, J., Suntzeff, N. B., Schommer, R. A., \& Avilés, R. 1995, AJ, 109,1

Hamuy, M., Phillips, M. M., Schommer, R. A., Suntzeff, N.B., Maza, J., \& Avilés, R. 1996a, AJ, 112,2391

Hamuy, M., Phillips, M. M., Suntzeff, N. B., Schommer, R. A., Maza, J., \& Avilés, R. 1996b, AJ, 112,2398

Hamuy, M., Phillips, M. M., Suntzeff, N. B., Schommer, R. A., Maza, J., Smith, R. C., Lira P., \& Avilés, R. 1996c, AJ, 112, 2438

Harris, W. E., Fitzgerald, H. P., \& Reed, B. C. 1981, PASP, 93507

Harness, R.P., and Wheeler, J.C. 1990, in Supernovae, ed. Petschek, A.G., New York: Springer-Verlag, p. 1.

Jacoby, G. H., \& Pierce, M. J. 1996, AJ, 112, 723

Jeffery, D. J., Leibundgut, B., Kirshner, R. P., Benetti, S., Branch, D., \& Sonneborn, G. 1992, ApJ, 397, 304

Johnson, H. L., 1963, in Basic Astronomical Data, edited by K. A. Strand, (Univ. Chicago Press, Chicago), p. 204

Kirshner, R., and Leibundgut, B. 1990, IAU Circ., No. 5039

Kirshner, R. P., Leibundgut, B, Foltz, C. B. , \& Pier, J. 1990, ApJ, 118, 502

Kirshner R. P., 1991, IAU Circ.No 5239

Kron, R. G. 1953, ApJ, 118, 502

La Franca, F., \& Goldschmidt, C. 1991, IAU Circ.No. 5239

Landolt, A. U. 1972, AJ, 78, 959

Landolt, A. U. 1992, AJ, 104, 340

Leibundgut, B. 1988, Ph.D. Thesis, Universitat Basel

Leibundgut, B., Kirshner, R. P., Filippenko, A. V, Shields, J. C., Foltz, C. B., Phillips, M. M., \& Sonneborn, G. 1991, ApJ, 371, 23

Lira P. 1996, Mc.S. thesis, Universidad de Chile

Madore, B.F., and Freedman, W.L. 1991, PASP, 103, 933

Maza, J., Hamuy, M., Phillips, M. M., Suntzeff, N. B., \& Avilés R. 1994, ApJ, 424, L107 
Mazzali, P. A., Danziger, I. J., \& Turatto, M. 1995, å, 297, 509

Maury, A. 1990, IAU Circ., No. 5039

Meikle, W. P. S., Cumming, R. J., Geballe, T. R., Lewis, J. R., Walton, N. A., Balcells, M., Cimatti, A., Croom, S. M., Dhillon, V. S., Economou, F., Jenkins, C. R., Knapen, J. H., Meadows, V. S., Morris, P. W., Perez-Fournon, I., Shanks, T., Smith, L. J., Tanvir, N. R., Veilleux, S., Vilchez, J., Wall, J. V., \& Lucey, J. R. 1996, MNRAS, 281, 263

Meyer, D.M., \& Roth, K.C. 1991, ApJ, 383, L41

Nomoto, K., Thielemann, F.-K., \& Yokoi, K. 1984, ApJ, 286, 644

Nugent, P., Phillips, M. M., Baron, E., Branch, D., \& Hauschildt, P. 1995, ApJ, 455, L147

Olsen, E.H. 1973, A\&AS, 54, 55

Pierce, M. J., \& Jacoby, G. H. 1995, AJ, 110, 2885

Phillips, M. M., Phillips, A. C., Heathcote, S. R., Blanco, V. M., Geisler, D., Hamilton, D., Suntzeff, N. B., Jablonski, F. J., Steiner, J. E., Cowley, A. P. et al. 1987, PASP, 99, 592

Phillips, M. M. 1993, ApJ, 413, L105

Phillips, M. M., Suntzeff, N. B., Hamuy, M., Leinbundgut, B., Kirshner, R. P., \& Foltz, C. B. 1992, AJ, 103, 1632

Phillips, M. M., \& Hamuy, M. 1991, IAU Circ.No. 5251

Pollas, C. 1990, IAU Circ.No. 5040

Pskovskii, Y. P. 1977, Soviet Astr, 21, 675

Pskovskii, Y. P. 1984, Soviet Astr., 28, 658

Qiao, Q.-Y., Wu, H., Wei, J.-Y., \& Li, W.-D. 1997a, IAU Circ.6623

Qiao, Q.-Y., Wu, H., Wei, J.-Y., \& Li, W.-D. 1997b, IAU Circ., 6642

Riess, A. G., Press, W. H., \& Kirshner, R. P. 1995, ApJ, 438, L17

Riess, A. G., Press, W. H., \& Kirshner, R. P. 1996, ApJ, 473, 88

Ruiz-Lapuente, P., Cappellaro, E., Turatto, M., Gouiffes, C., Danziger, I. J., Della Valle, M., \& Lucy, L. B. 1992, ApJ, 387, L33

Rust, B.W., "The Use of Supernovae Light Curves for Testing the Expansion Hypothesis and Other Cosmological Relations," Ph.D. thesis, Univ. Illinois. 
Saha, A., Sandage, A., Labhardt, L., Tammann, G. A., Macchetto, F. D., \& Panagia, N. 1996, ApJS, 107, 693

Sandage, A., \& Tammann, G. A. 1993, ApJ, 415, 1

Sandage, A., Saha, A., Tammann, G. A., Labhardt, L., Panagia, N., \& Maccheto, F. D. 1996, ApJ, 460, L15

Savage, B. D., \& Mathis, J. S. 1979, ARA\&A, 17, 73

Schaefer, B. 1996, AJ, 111, 1668

Schmidt, B. P., Kirshner, R. P., Leibundgut, B., Wells, L. A., Porter, A. C., Ruiz-Lapuente, P., Challis, P., \& Filippenko, A. V. 1994, ApJ, 434, L19

Serkowski, K. 1970, PASP, 82, 908

Shigeyama, T., Nomoto, K., Yamaoka, H., \& Thielemann, F. 1992, ApJ, 386, L13

Silbermann, N.A., Harding, P., Madore, B.F., Kennicutt, R.C., Freedman, W.L., Mould, J.R., Stetson, P.B., Saha, A., Bresolin, F., Turner, A., Ferrarese, L., Ford, H., Gibson, B., Rawson, D., Graham, J., Han, M., Hoessel, J.G., Hill, R.J., Huchra, J., Macri, J., Hughes, S.M.G., Illingworth, G.D., Kelson, D., Phelps, R., \& Sakai, S. 1996, In “ The STScI May Symp.; The Extragalactic Distance Scale" STScI preprint of poster papers, p. 67

Spyromilio, J., Meikle, W. P., Allen, D. A., \& Graham, J. R. 1992, MNRAS, 258, 53

Stetson, P. B. 1987, PASP, 99, 191

Suntzeff, N. B. 1995, in Supernovae and Supernova Remnants, IAU Colloquium 145, edited by R. McCray (Cambridge University Press, Cambridge), p. 41

Tammann, G. A., \& Sandage, A., 1995, ApJ, 452, 16

Tanvir, N. R., Shanks, T., Ferguson, H. C., Robinson, D. T. R. 1995, Nature, 377, 27

Waagen, E. et al. 1991, IAU Circ.No. 5239

Walker, G.A.H., Andrews, D., Hill, G., Morris, S.C., Smyth, W. and White, J., 1970, D.A.O. Publications XIII, Pt. 17, 415

Wells, L. A., Phillips, M. M., Suntzeff, B., Heathcote, S. R., Hamuy, M., Navarrete, M., Fernandez, M., Weller, W. G., Schommer, R. A., Kirshner, R. P., Leibundgut, B., Willner, S. P., Peletier, S. P., Schlegel, E. M., Wheeler, J. C., Harkness, R. P., Bell, D. J., Matthews, J. M., Filippenko, A. V., Shields, J. C., Richmond, W., Jewitt, D., Luu, J., Tran, H. D., Appleton, P. N., Robson, E. I., Tyson, J. A., Guhathakurta, P., Eder, J. A., Bond, H. E., Potter, M., Veilleux, S., Porter, A. C., Humphreys, R. M., Janes, K. A., Williams, T. B., 
Costa, E., Ruiz, M. T., Lee, J. T., Lutz, J. H., Rich, R. M., Winkler, P. F., \& Tyson, N. D. 1994, AJ, 108, 2233

Wheeler, J.C., \& Smith, V.V. 1991, IAU Circ., 5256

Woosley, S.E., Pinto, P.A., Hartmann, D. 1989, ApJ, 346, 395

Yamaoka, H., Nomoto, K., Shigeyama, T., \& Thielemann, F. 1992, ApJ, 393, L55 
Fig. 1.- SN 1990N in NGC 4639. The local standards listed in Table 3 are marked. This $R$ image was taken at the CTIO $0.9 \mathrm{~m}$ telescope on 13 March 1994 . The field is $6.8^{\prime}$ on a side. Star 14 does not appear on this chart. It is located at $(12: 42: 47.0,+13: 17: 52$, J2000) on the Digitized Sky Survey.

Fig. 2.- SN 1991T in NGC 4527. The local standards listed in Table 4 are marked. This $R$ image was taken at the CTIO $0.9 \mathrm{~m}$ telescope on 14 March 1994 . The field is $6.8^{\prime}$ on a side.

Fig. 3.- $U B V R I$ light curves of SN 1990N.

Fig. 4.- UBVRI light curves of SN 1991T.

Fig. 5.- Comparison of the $B$ and $V$ photometry of SN $1990 \mathrm{~N}$ given in this paper (circles) with the preliminary photometric reductions of the CTIO data by Leibundgut et al. (1991)(triangles).

Fig. 6.- Comparison of our $B$ and $V$ light curves of SN 1991T (filled circles) with the preliminary photometric reductions of the CTIO data published by Phillips et al. (1992) (triangle). The $V$ magnitudes from the Van Vleck Observatory study Ford et al. (1993)] are plotted as squares.

Fig. 7.- BV light curves of SN 1990N compared with the SN Ia template curves determined by Leibundgut (1988). The abscissa is plotted in units of days as JD-2440000.

Fig. 8.- $B V$ light curves of SN $1991 \mathrm{~T}$ compared with the SN Ia template curves determined by Leibundgut (1988) The abscissa is plotted in units of days as JD-2440000. The data appear to fall from maximum more slowly than the template curves..

Fig. 9.- Late-time $B V$ light curves for SN 1991T. Photometry from CTIO (filled circles) and from KPNO (triangle) are shown. An estimated decline rate for type Ia supernovae based on the Pskovskii $\gamma$ parameter is also plotted.

Fig. 10.- Observed $B-V, B-I$ and $B-R$ color evolution for SN 1990N (open circles) and SN $1991 \mathrm{~T}$ (filled circles) as a function of time since $B_{\max }$.

Fig. 11.- Observed $V-R, V-I$ and $R-I$ color evolution of SN 1990N (open circles) and SN $1991 \mathrm{~T}$ (filled circles) as a function of time since $B_{\max }$.

Fig. 12.- Observed $B-V$ versus $R-I$ color-color curves for SN 1990N (circle) and SN 1991T (filled circles). The numbers indicate the time elapsed, in days, since $B$ maximum. 
TABLE 1. Observation Log for SN 1990N.

TABLE 2. Observation Log for SN 1991T.

TABLE 3. Photometric sequence for SN 1990N.

TABLE 1. Photometric sequence for SN 1991T.

TABLE 5. SN 1990N photometry.

TABLE 6. SN 1991T photometry.

TABLE 01. Peak photometric magnitudes.

TABLE 8. Pskovskii and Phillips parameters. 


\section{SN 1990N}

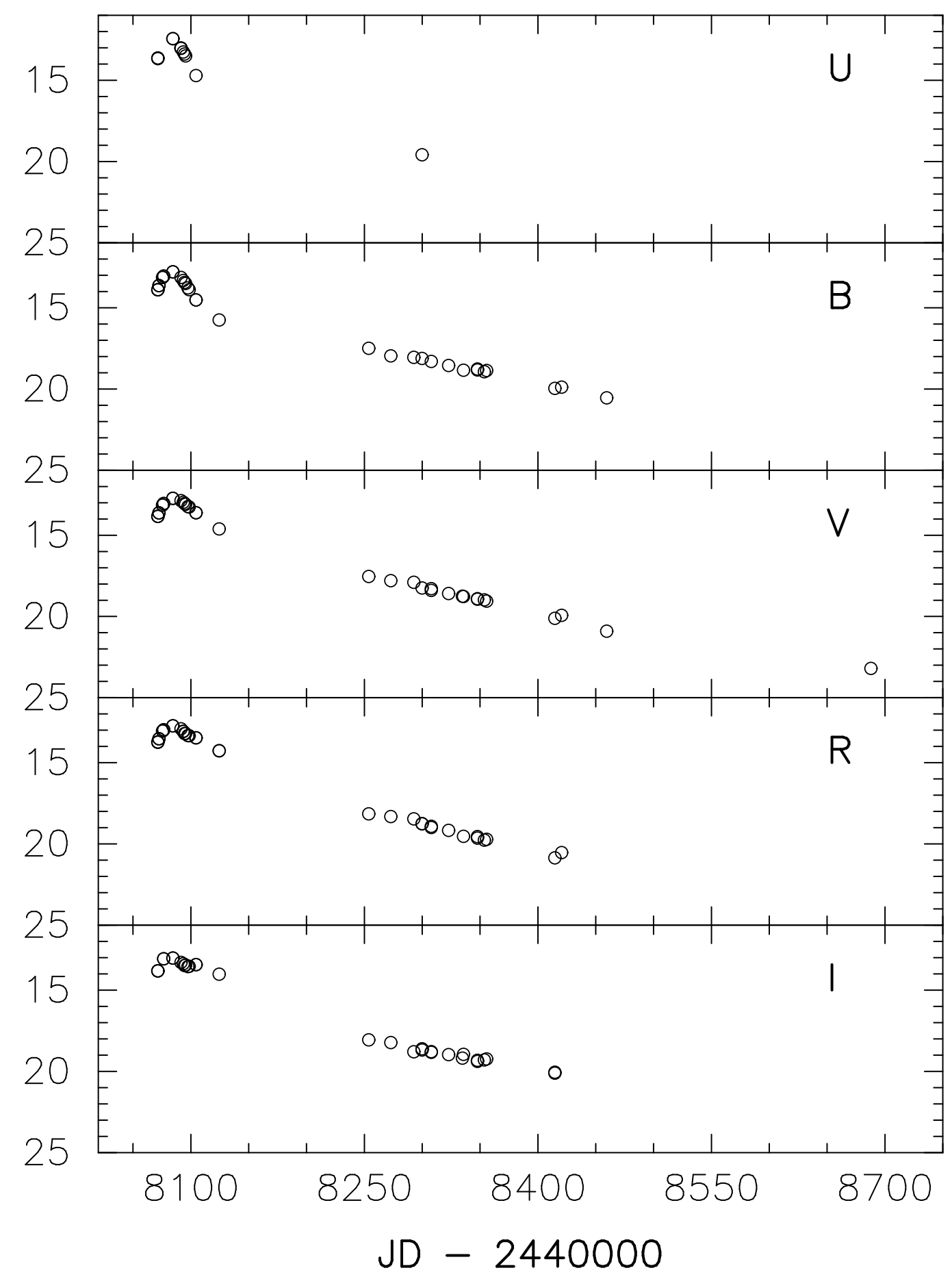

Lira et al. Figure 3 
SN $1991 \mathrm{~T}$

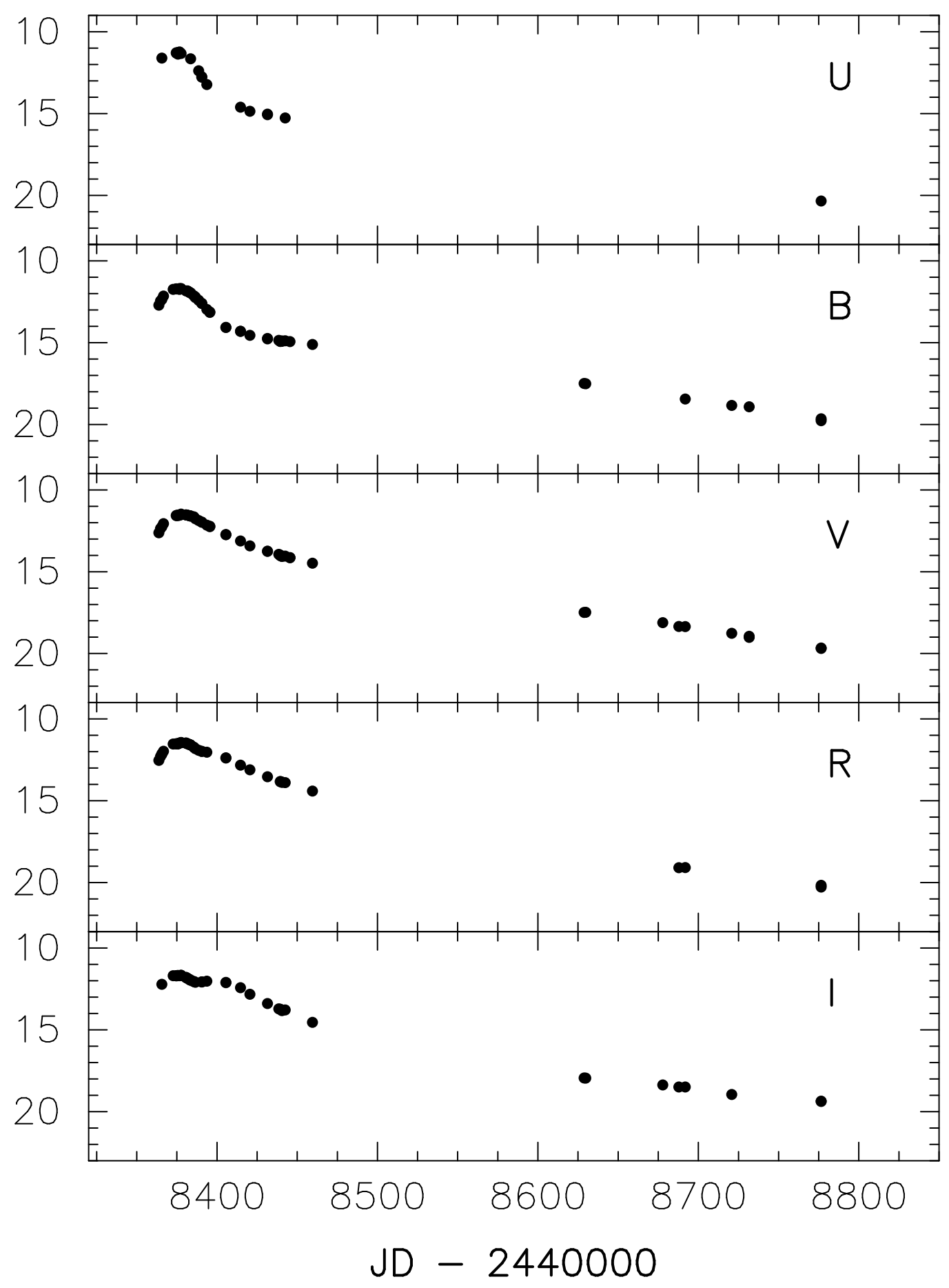

Lira et al. Figure 《 


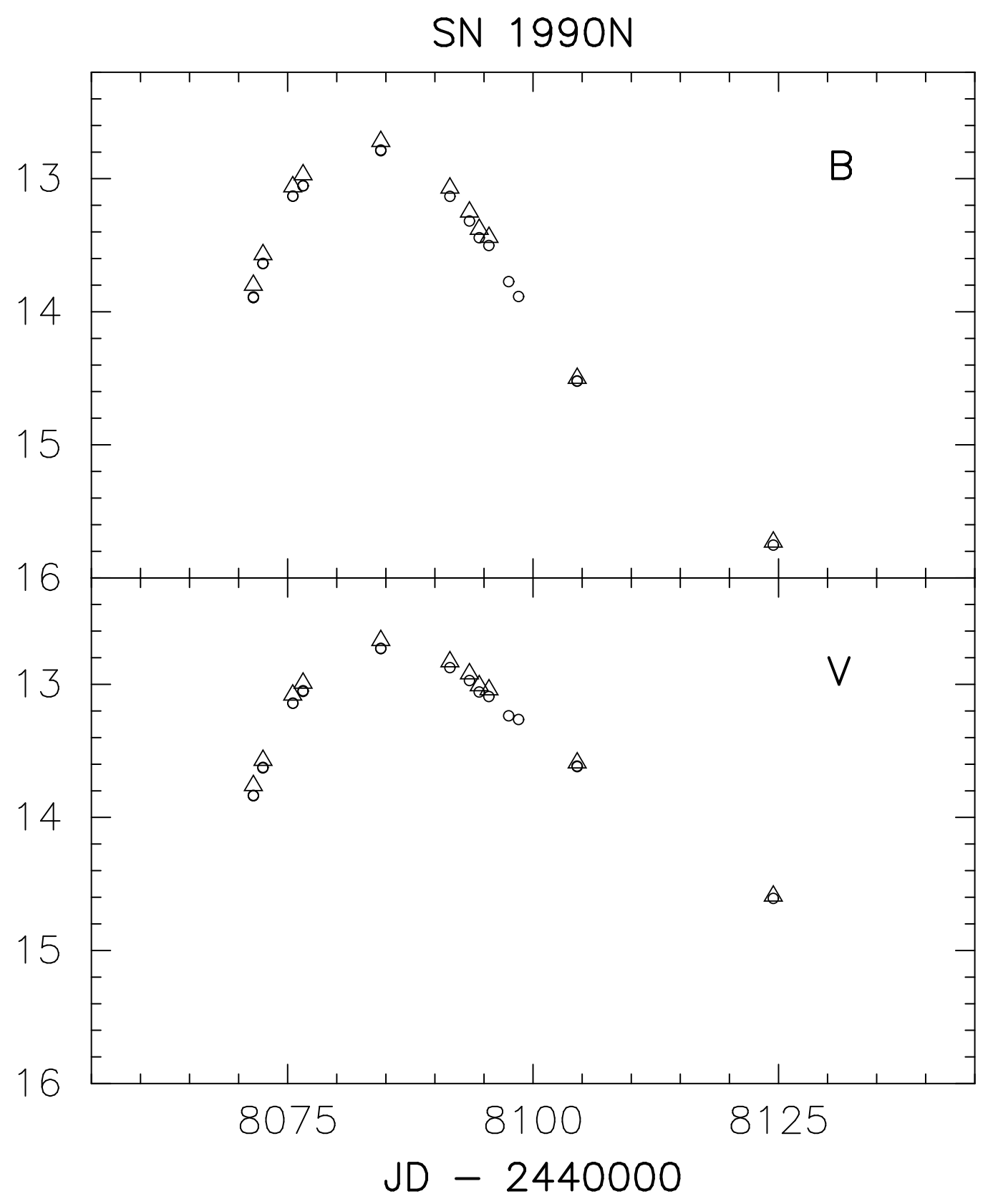

Lira et al. Figure 5 


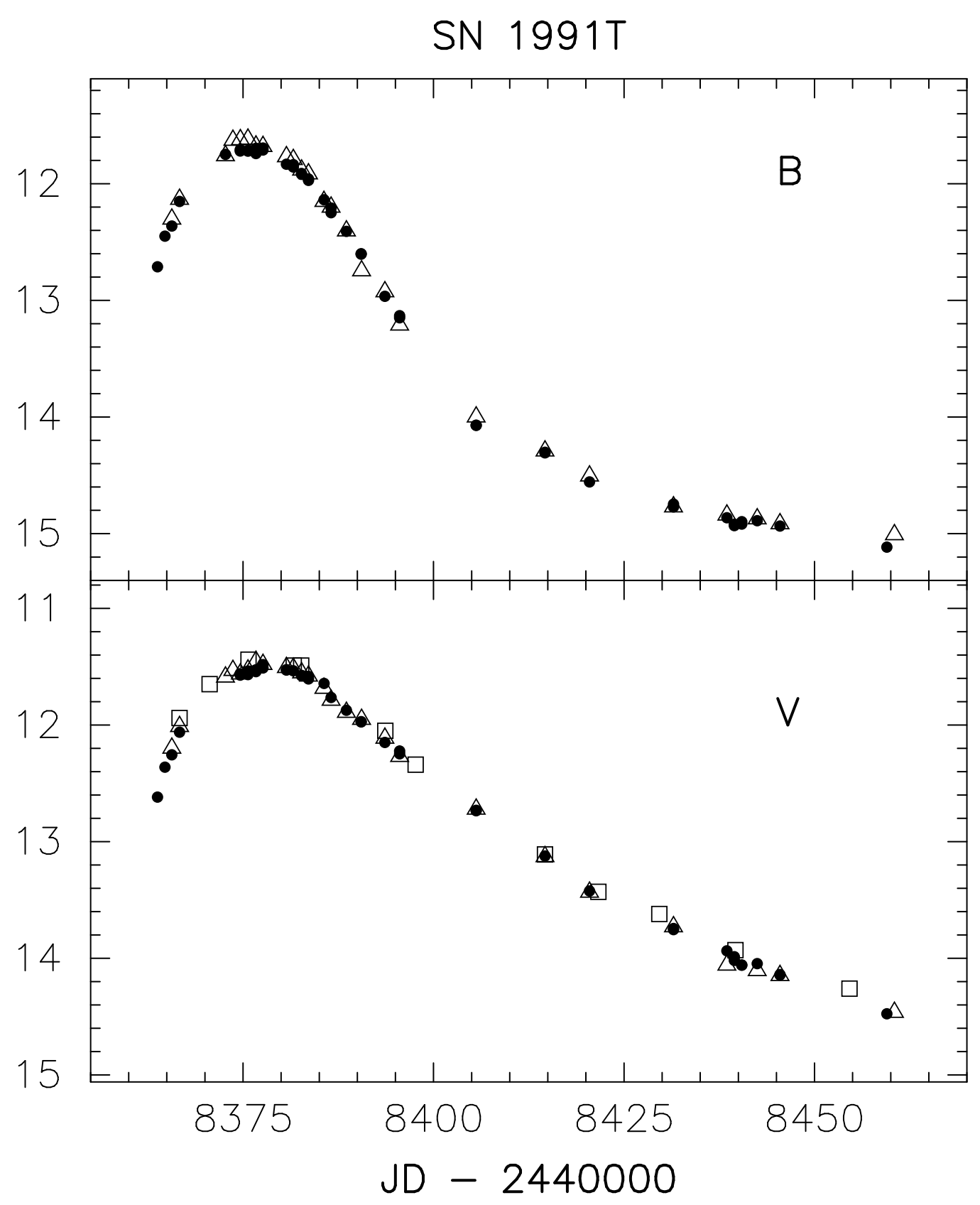

Lira et al. Figure 6 


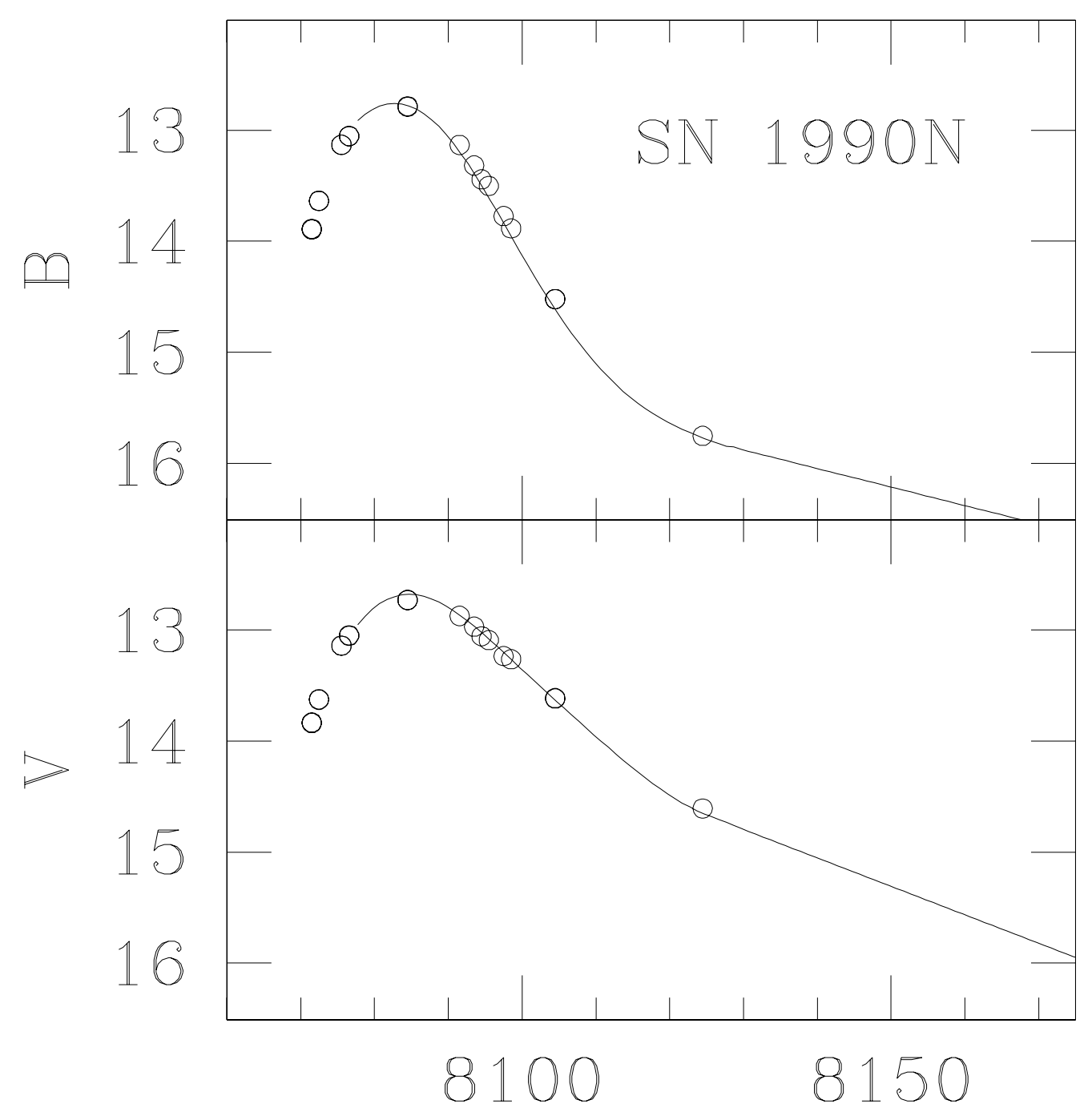

Lira et al. Figure 0 


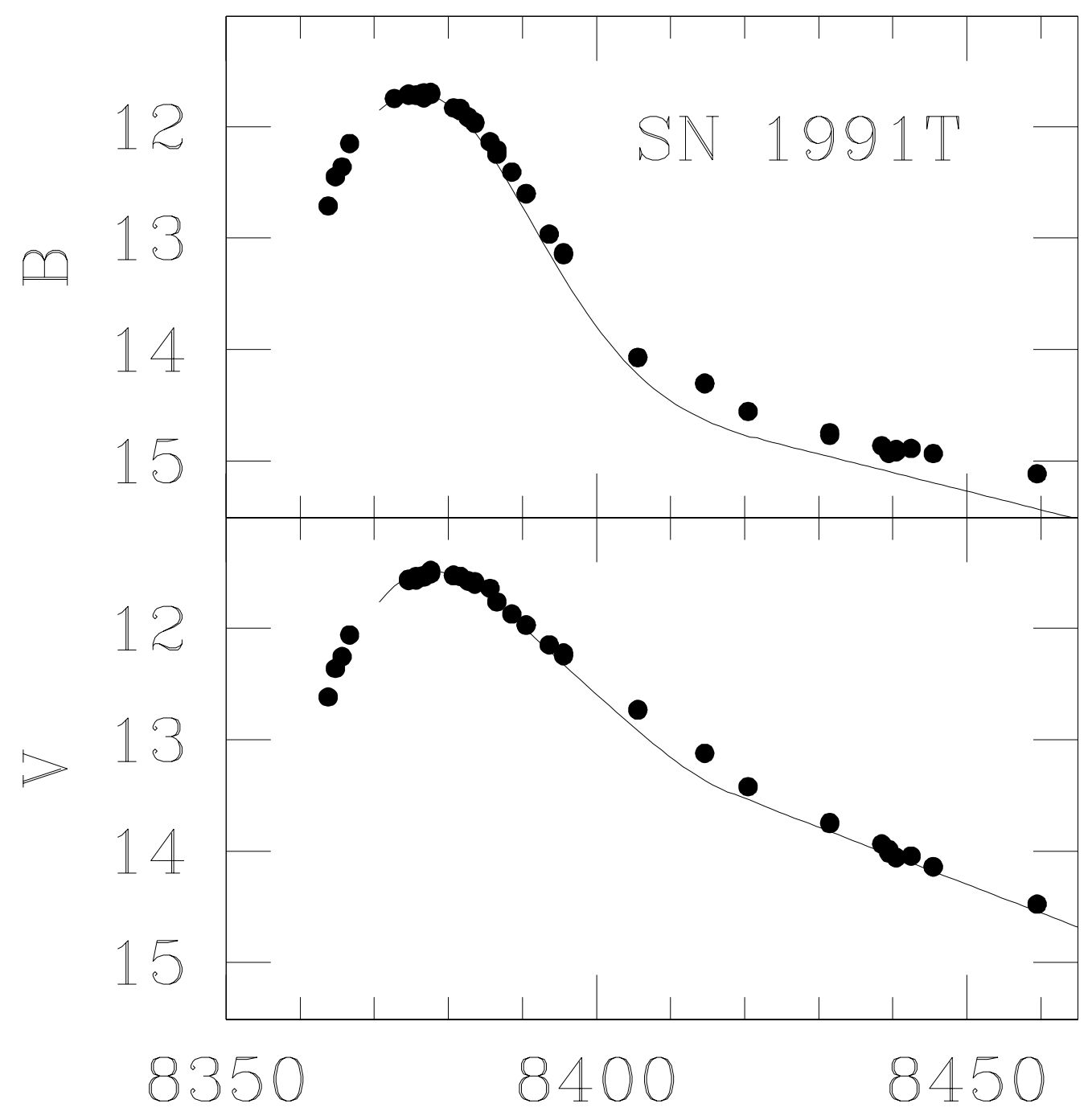

Lira et al. Figure 8 


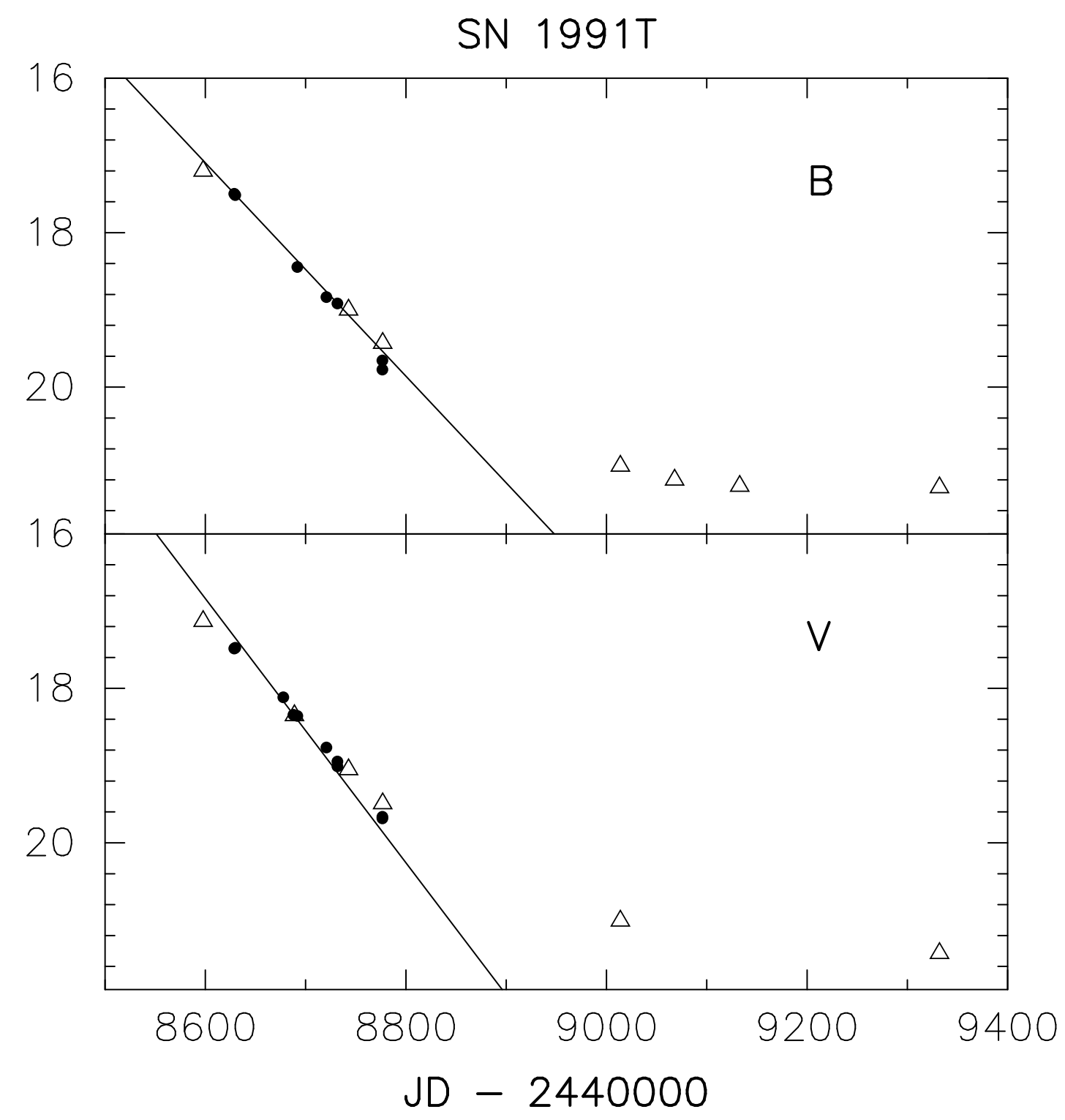

Lira et al. Figure 9 


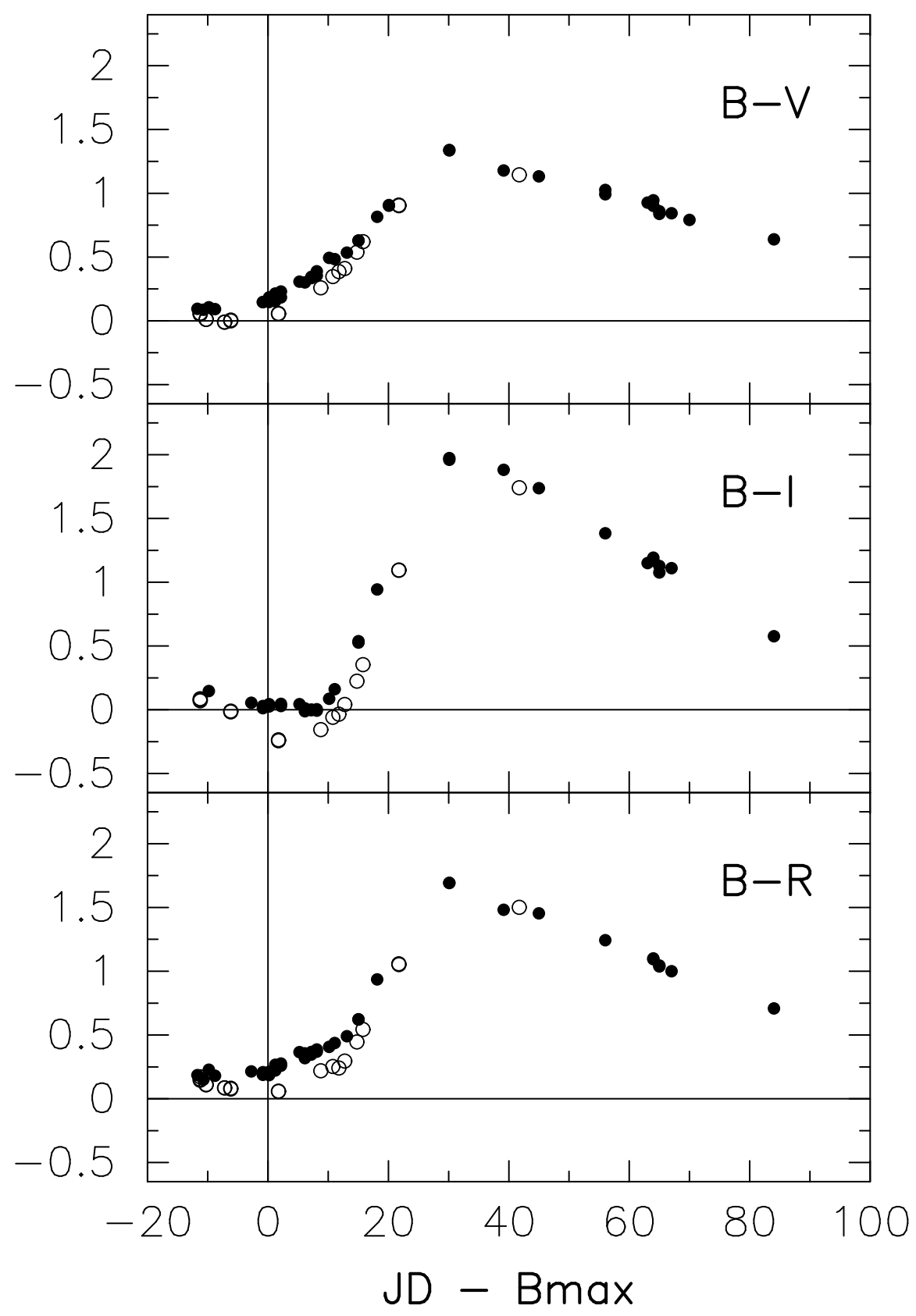

Lira et al. Figure 10 


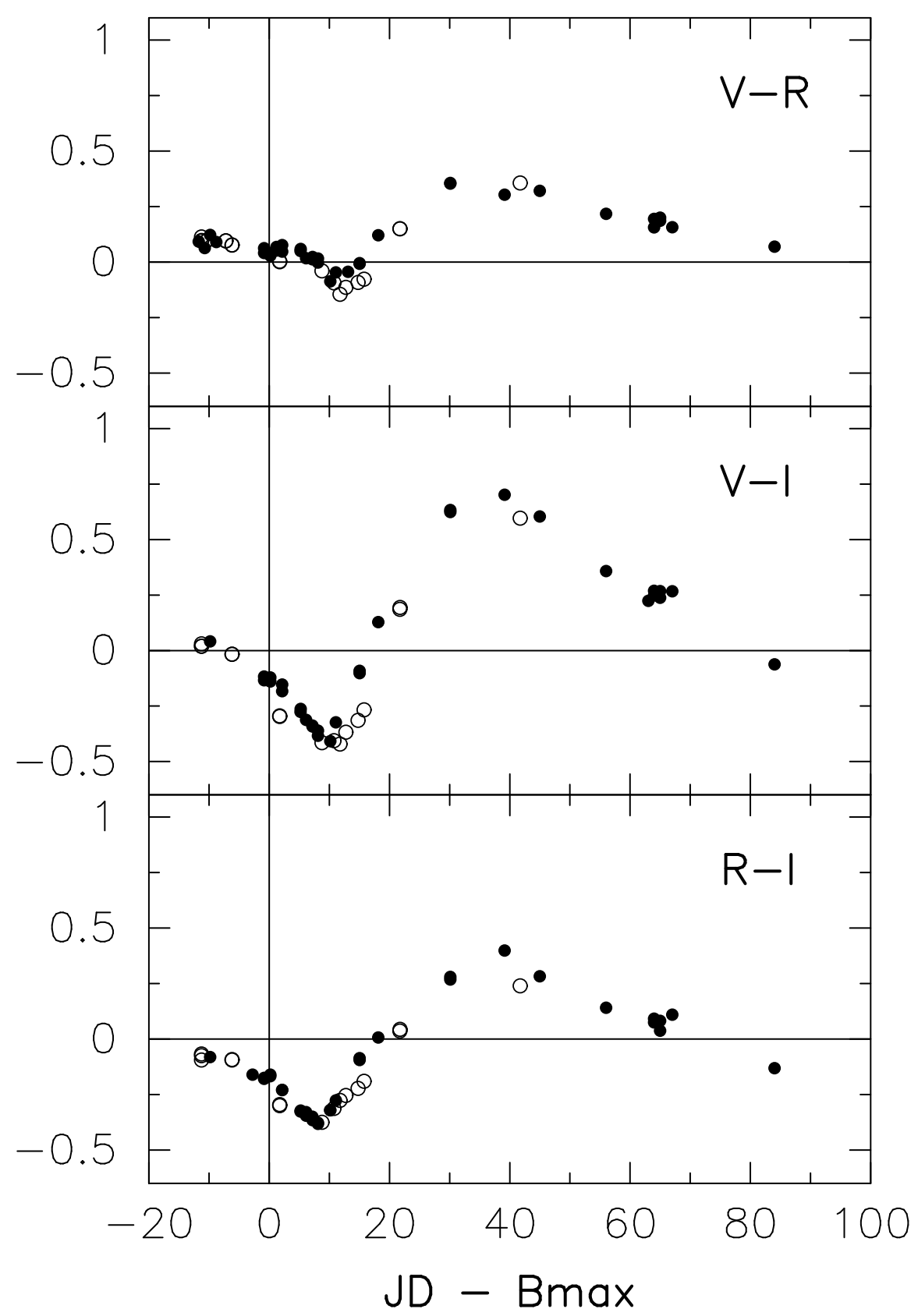

Lira et al. Figure 11 


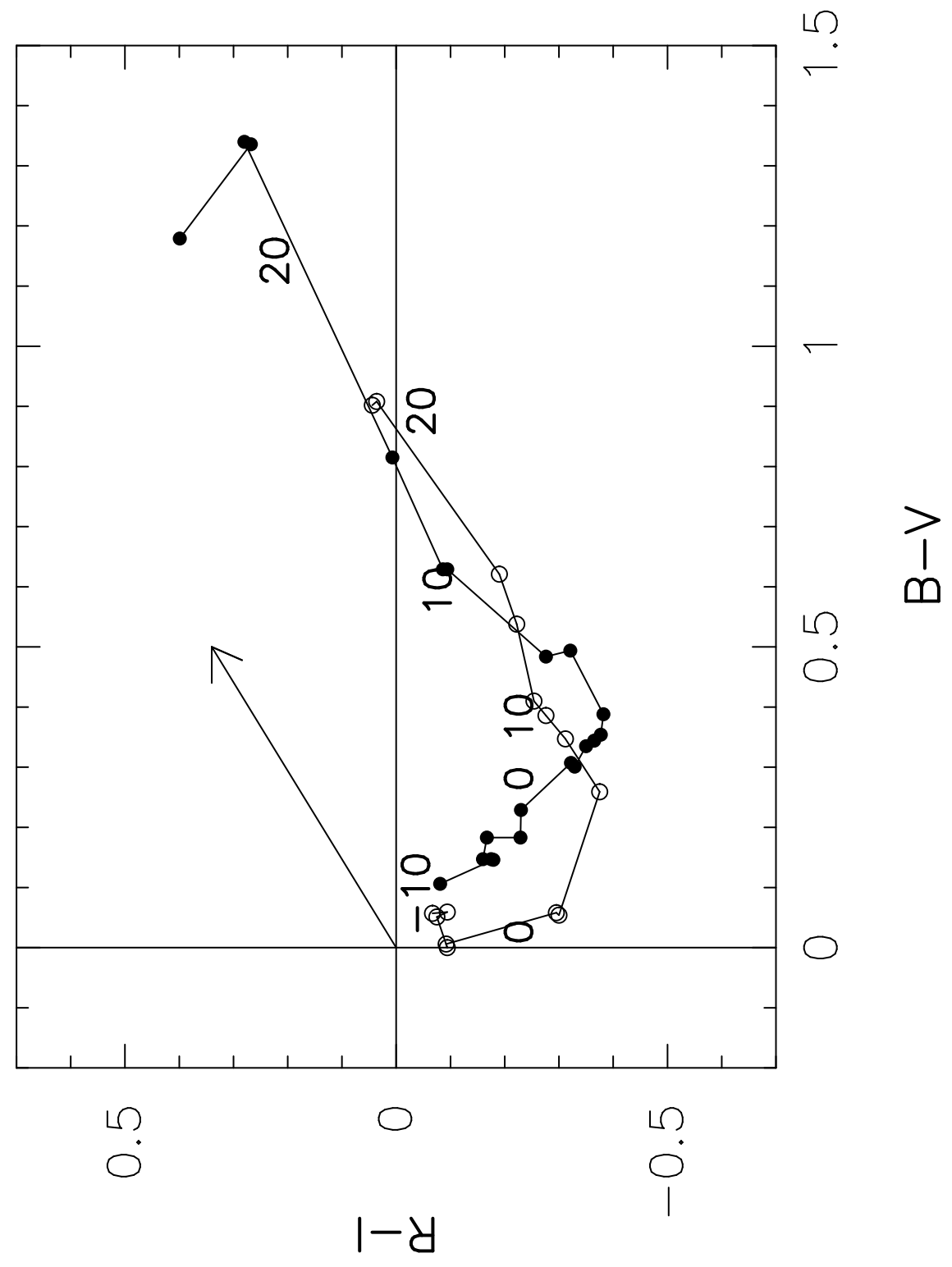

Lira et al. Figure 12 
TABLE 1. Observation log for SN 1990N.

\begin{tabular}{lllll}
\hline \hline Date(UT) & Telescope & \multicolumn{1}{c}{ Observers } & Filters & Detector \\
\hline 1990 Jun 29 & $4.0 \mathrm{~m}$ & Navarrete & $U B V R I$ & TI2 \\
1990 Jul 3 & $0.91 \mathrm{~m}$ & Rehner, Siciliano & $B V R$ & TEK4 \\
1990 Jul 4 & $0.91 \mathrm{~m}$ & Rehner, Siciliano & $B V R$ & TEK4 \\
1990 Jul 4 & $0.91 \mathrm{~m}$ & Suntzeff & $B V R I$ & TEK4 \\
1990 Jul 12 & $0.91 \mathrm{~m}$ & Wells & $U B V R I$ & TEK4 \\
1990 Jul 19 & $0.91 \mathrm{~m}$ & Tyson, Rich & $U B V R I$ & TI2 \\
1990 Jul 20 & $0.91 \mathrm{~m}$ & Tyson, Rich & $U B V R I$ & TI2 \\
1990 Jul 21 & $0.91 \mathrm{~m}$ & Tyson, Rich & $U B V R I$ & TI2 \\
1990 Jul 22 & $0.91 \mathrm{~m}$ & Tyson, Rich & $U B V R I$ & TI2 \\
1990 Jul 25 & $0.91 \mathrm{~m}$ & Williams & $B V R I$ & TEK4 \\
1990 Jul 26 & $0.91 \mathrm{~m}$ & Williams & $B V R I$ & TEK4 \\
1990 Aug 1 & $0.91 \mathrm{~m}$ & Suntzeff & $U B V R I$ & TI3 \\
1990 Aug 21 & $0.91 \mathrm{~m}$ & Elias & $B V R I$ & TI3 \\
1990 Dec 28 & $0.91 \mathrm{~m}$ & Baganoff & $B V R I$ & TI3 \\
1991 Jan 16 & $0.91 \mathrm{~m}$ & González & $B V R I$ & TI3 \\
1991 Feb 5 & $0.91 \mathrm{~m}$ & Navarrete & $B V R I$ & TI2 \\
1991 Feb 12 & $4.0 \mathrm{~m}$ & Navarrete & $U B V R I$ & TEK1 \\
1991 Feb 20 & $0.91 \mathrm{~m}$ & Navarrete & $B V R I$ & TEK4 \\
1991 Mar 7 & $0.91 \mathrm{~m}$ & Suntzeff & $B V R I$ & TI3 \\
1991 Mar 19 & $0.91 \mathrm{~m}$ & Roth & $V I$ & Thomson \\
1991 Mar 20 & $0.91 \mathrm{~m}$ & Suntzeff & $B V R I$ & TI3 \\
1991 Apr 1 & $0.91 \mathrm{~m}$ & Crotts & $U B V R I$ & TEK1 \\
1991 Apr 7 & $0.91 \mathrm{~m}$ & Ugarte & $B V R I$ & TEK2 \\
1991 Apr 9 & $4.0 \mathrm{~m}$ & Navarrete & $B V R I$ & TEK1 \\
1991 Jun 7 & $4.0 \mathrm{~m}$ & Navarrete & $B V R I$ & TI2 \\
1991 Jun 13 & $0.91 \mathrm{~m}$ & Navarrete & $B V R$ & TEK4 \\
1991 Jul 23 & $0.91 \mathrm{~m}$ & Dey & $B V$ & TEK2 \\
1992 Mar 6 & $4.0 \mathrm{~m}$ & Suntzeff, Navarrete & $V R$ & TEK2 \\
\hline \hline
\end{tabular}


TABLE 2. Observation log for SN 1991T.

\begin{tabular}{|c|c|c|c|c|}
\hline Date(UT) & Telescope & Observers & Filters & Detector \\
\hline 1991 Apr 17 & $0.91 \mathrm{~m}$ & Hibbard, Guhathakurta & $B V R$ & TEK4 \\
\hline 1991 Apr 18 & $0.91 \mathrm{~m}$ & Hibbard, Guhathakurta & $B V R$ & TEK4 \\
\hline 1991 Apr 19 & $0.91 \mathrm{~m}$ & Schommer & $U B V R I$ & TI3 \\
\hline 1991 Apr 20 & $0.91 \mathrm{~m}$ & Baldwin & $B V R$ & TI3 \\
\hline 1991 Apr 26 & $0.91 \mathrm{~m}$ & Schommer & $B V R I$ & TEK1 \\
\hline 1991 Apr 27 & $0.91 \mathrm{~m}$ & Schommer & $U B V I$ & TEK1 \\
\hline 1991 Apr 28 & $0.91 \mathrm{~m}$ & Wells & $U B V R I$ & TI3 \\
\hline 1991 Apr 29 & $0.91 \mathrm{~m}$ & Wells & $U B V R I$ & TI3 \\
\hline 1991 Apr 30 & $0.91 \mathrm{~m}$ & Wells & $U B V R I$ & TI3 \\
\hline 1991 May 1 & $0.91 \mathrm{~m}$ & Wells & $U B V R I$ & TEK4 \\
\hline 1991 May 4 & $0.91 \mathrm{~m}$ & Williger & $B V R I$ & TEK4 \\
\hline 1991 May 5 & $0.91 \mathrm{~m}$ & Williger & $B V R I$ & TEK4 \\
\hline 1991 May 6 & $0.91 \mathrm{~m}$ & Williger & $B V R I$ & TEK4 \\
\hline 1991 May 7 & $0.91 \mathrm{~m}$ & Navarrete & $U B V R I$ & TEK4 \\
\hline 1991 May 9 & $4.0 \mathrm{~m}$ & Navarrete & $B V R I$ & TEK1 \\
\hline 1991 May 10 & $0.91 \mathrm{~m}$ & Walker & $B V R I$ & TI3 \\
\hline 1991 May 12 & $0.91 \mathrm{~m}$ & Tyson & $U B V R I$ & TEK4 \\
\hline 1991 May 14 & $0.91 \mathrm{~m}$ & Wells & $U B V R I$ & TI3 \\
\hline 1991 May 17 & $0.91 \mathrm{~m}$ & González & $U B V R I$ & TI3 \\
\hline 1991 May 19 & $0.91 \mathrm{~m}$ & Layden & $B V$ & TI3 \\
\hline 1991 May 29 & $0.91 \mathrm{~m}$ & Williger & $B V R I$ & TEK2 \\
\hline 1991 Jun 7 & $4.0 \mathrm{~m}$ & Navarrete & $U B V R I$ & TI2 \\
\hline 1991 Jun 13 & $0.91 \mathrm{~m}$ & Navarrete & $U B V R I$ & TEK4 \\
\hline 1991 Jun 24 & $0.91 \mathrm{~m}$ & Schommer & $U B V R I$ & TEK2 \\
\hline 1991 Jul 1 & $0.91 \mathrm{~m}$ & Seitzer & $B V I$ & TEK1 \\
\hline $1991 \mathrm{Jul} 2$ & $0.91 \mathrm{~m}$ & Schommer & $B V R I$ & TI3 \\
\hline 1991 Jul 3 & $0.91 \mathrm{~m}$ & Williams & $B V R I$ & TI3 \\
\hline 1991 Jul 5 & $0.91 \mathrm{~m}$ & Navarrete & $U B V R I$ & TI3 \\
\hline 1991 Jul 8 & $0.91 \mathrm{~m}$ & Siciliano & $B V$ & TEK2 \\
\hline 1991 Jul 22 & $0.91 \mathrm{~m}$ & Dey & $B V R I$ & TEK2 \\
\hline 1992 Jan 7 & $0.91 \mathrm{~m}$ & Schommer & $B V I$ & TEK2 \\
\hline $1992 \operatorname{Jan} 8$ & $0.91 \mathrm{~m}$ & Schommer & $B V I$ & TEK2 \\
\hline 1992 Feb 25 & $0.91 \mathrm{~m}$ & Siciliano & $V I$ & TEK1 \\
\hline 1992 Mar 6 & $4.0 \mathrm{~m}$ & Suntzeff, Navarrete & $B V R I$ & TEK1 \\
\hline 1992 Mar 10 & $0.91 \mathrm{~m}$ & Hamuy & $B V R I$ & TEK4 \\
\hline 1992 Apr 8 & $0.91 \mathrm{~m}$ & Kim & $B V I$ & TEK2 \\
\hline 1992 Apr 19 & $0.91 \mathrm{~m}$ & Schommer & $B V$ & TEK1 \\
\hline 1992 Jun 4 & $0.91 \mathrm{~m}$ & Wells & $U B V R I$ & TEK4 \\
\hline
\end{tabular}


TABle 3. Photometric sequence for SN 1990N.

\begin{tabular}{rrrccccr}
\hline \hline Star $^{\mathrm{a}}$ & $\mathrm{n}$ & $\mathrm{m}$ & $\begin{array}{c}V \\
\mathrm{mag}(\mathrm{m.e.})\end{array}$ & $\begin{array}{c}B-V \\
\mathrm{mag}(\mathrm{m.e.})\end{array}$ & $\begin{array}{c}V-R \\
\operatorname{mag}(\mathrm{m.e.})\end{array}$ & $\begin{array}{c}R-I \\
\operatorname{mag}(\mathrm{m.e.})\end{array}$ & $\begin{array}{c}U-B \\
\operatorname{mag}(\mathrm{m} . \mathrm{e} .)\end{array}$ \\
\hline 1 & 5 & 9 & $15.728(005)$ & $1.076(009)$ & $0.646(006)$ & $0.512(011)$ & $1.002(026)$ \\
2 & 5 & 8 & $17.810(003)$ & $0.659(007)$ & $0.414(004)$ & $0.457(009)$ & $0.013(012)$ \\
3 & 7 & 13 & $18.514(004)$ & $0.702(005)$ & $0.453(005)$ & $0.457(006)$ & $0.013(021)$ \\
4 & 6 & 12 & $17.118(004)$ & $0.623(006)$ & $0.379(005)$ & $0.362(006)$ & $0.074(009)$ \\
5 & 7 & 13 & $18.846(005)$ & $0.730(007)$ & $0.435(006)$ & $0.408(009)$ & $0.280(033)$ \\
6 & 7 & 13 & $18.622(005)$ & $0.519(008)$ & $0.333(006)$ & $0.362(009)$ & $-0.086(024)$ \\
7 & 6 & 12 & $17.707(003)$ & $0.735(006)$ & $0.414(004)$ & $0.402(005)$ & $0.068(018)$ \\
8 & 3 & 6 & $17.626(003)$ & $1.468(006)$ & $0.940(004)$ & $0.933(005)$ & $1.256(092)$ \\
9 & 7 & 12 & $19.543(005)$ & $0.479(010)$ & $0.377(006)$ & $0.466(010)$ & $-0.129(021)$ \\
10 & 7 & 12 & $19.573(010)$ & $0.863(026)$ & $0.539(013)$ & $0.705(014)$ & $-0.269(074)$ \\
11 & 5 & 9 & $16.580(004)$ & $0.615(007)$ & $0.385(006)$ & $0.351(006)$ & $0.040(008)$ \\
12 & 7 & 13 & $19.140(007)$ & $1.484(023)$ & $1.104(013)$ & $1.509(014)$ & $0.601(066)$ \\
13 & 5 & 9 & $18.467(010)$ & $1.285(021)$ & $0.860(011)$ & $0.871(006)$ & $0.941(062)$ \\
14 & 4 & 9 & $16.602(005)$ & $1.455(007)$ & $0.955(009)$ & $0.996(009)$ & $1.072(031)$ \\
15 & 3 & 6 & $13.392(001)$ & $0.607(002)$ & $0.356(002)$ & $0.341(003)$ & $0.031(200)$ \\
\hline \hline
\end{tabular}

${ }^{\text {a See Figure } 1 .}$ 
TABLE 4. Photometric sequence for SN 1991T.

\begin{tabular}{crrrcccr}
\hline \hline Star $^{\mathrm{a}}$ & $\mathrm{n}$ & $\mathrm{m}$ & $\begin{array}{c}V \\
\text { mag (m.e.) }\end{array}$ & $\begin{array}{c}B-V \\
\text { mag (m.e.) }\end{array}$ & $\begin{array}{c}V-R \\
\text { mag (m.e.) }\end{array}$ & $\begin{array}{c}R-I \\
\text { mag (m.e.) }\end{array}$ & $\begin{array}{c}U-B \\
\text { mag (m.e.) }\end{array}$ \\
\hline 1 & 6 & 10 & $15.172(009)$ & $0.908(010)$ & $0.523(011)$ & $0.483(007)$ & $0.647(022)$ \\
$2^{\mathrm{b}}$ & 7 & 11 & $15.289(009)$ & $1.468(011)$ & $1.006(012)$ & $1.057(011)$ & $1.234(033)$ \\
3 & 6 & 8 & $14.367(014)$ & $1.338(026)$ & $0.694(014)$ & $0.657(006)$ & $1.257(053)$ \\
4 & 6 & 10 & $15.164(006)$ & $0.768(006)$ & $0.445(008)$ & $0.393(009)$ & $0.709(019)$ \\
5 & 3 & 6 & $14.900(007)$ & $0.757(007)$ & $0.442(009)$ & $0.408(006)$ & $0.380(005)$ \\
6 & 3 & 6 & $16.744(005)$ & $1.548(009)$ & $0.994(009)$ & $1.092(014)$ & $1.068(008)$ \\
$7^{\mathrm{c}}$ & 8 & 12 & $16.733(003)$ & $1.173(009)$ & $0.721(006)$ & $0.630(010)$ & $1.097(011)$ \\
$8^{\mathrm{c}}$ & 7 & 11 & $16.304(003)$ & $1.480(006)$ & $0.959(007)$ & $0.979(006)$ & $1.075(042)$ \\
$9^{\mathrm{c}}$ & 8 & 11 & $18.488(010)$ & $0.441(011)$ & $0.298(012)$ & $0.302(017)$ & $-0.241(014)$ \\
$\mathrm{G}^{\mathrm{d}}$ & 4 & 7 & $14.297(007)$ & $1.290(007)$ & $0.837(010)$ & $0.885(009)$ & $0.241(105)$ \\
\hline \hline
\end{tabular}

${ }^{\text {a See Figure } 2 .}$

${ }^{\mathrm{b}} \mathrm{Star} 2$ is also star 2 in Ford et al. (1993).

${ }^{\mathrm{c}} \mathrm{Stars}(7,8,9)$ are stars $(2,1,3)$ in Schmidt et al. (1994).

${ }^{\mathrm{d}} \mathrm{G}$ corresponds to the photometry of NGC 4527 nucleus. 
TABLE 5. SN 1990N photometry.

\begin{tabular}{|c|c|c|c|c|c|}
\hline $\begin{array}{c}\text { J.D. - } \\
2440000\end{array}$ & $\begin{array}{c}U \\
\operatorname{mag}(\text { m.e. })\end{array}$ & $\begin{array}{c}B \\
\operatorname{mag}(\text { m.e. })\end{array}$ & $\begin{array}{c}V \\
\operatorname{mag}(\text { m.e. })\end{array}$ & $\begin{array}{c}R \\
\operatorname{mag}(\text { m.e. })\end{array}$ & $\begin{array}{c}I \\
\text { mag (m.e.) }\end{array}$ \\
\hline 8071.50 & $13.645(026)$ & 13.894(017) & 13.837(017) & $13.740(013)$ & $13.807(015)$ \\
\hline 8071.50 & 13.625(026) & 13.893(017) & 13.834(017) & $13.721(013)$ & $13.815(016)$ \\
\hline 8071.50 & 13.671(026) & 13.888(017) & 13.837(017) & $13.742(013)$ & $13.817(016)$ \\
\hline 8072.47 & $\ldots$ & $13.638(017)$ & 13.628(017) & 13.529(013) & $\ldots$ \\
\hline 8072.48 & $\ldots$ & 13.636(017) & 13.624(017) & $13.525(013)$ & $\cdots$ \\
\hline 8075.53 & $\ldots$ & 13.131(017) & 13.141(017) & 13.046(013) & $\cdots$ \\
\hline 8075.53 & $\ldots$ & 13.132(017) & 13.142(017) & 13.046(013) & $\cdots$ \\
\hline 8076.56 & $\ldots$ & $13.053(017)$ & $13.053(017)$ & 12.976(013) & $13.070(015)$ \\
\hline 8076.56 & $\ldots$ & $13.054(017)$ & 13.048(017) & $12.972(013)$ & 13.064(015) \\
\hline 8084.49 & $12.438(026)$ & $12.790(017)$ & $12.732(017)$ & $12.731(013)$ & $13.026(015)$ \\
\hline 8084.49 & $12.446(026)$ & $12.784(017)$ & $12.730(017)$ & $12.727(013)$ & $13.027(015)$ \\
\hline 8091.53 & $13.018(026)$ & $13.133(017)$ & $12.874(017)$ & 12.914(013) & $13.289(016)$ \\
\hline 8091.53 & $13.032(026)$ & & & & \\
\hline 8091.53 & $13.014(026)$ & & & & \\
\hline 8093.51 & $13.257(026)$ & 13.318(017) & $12.971(017)$ & $13.065(013)$ & $13.377(016)$ \\
\hline 8093.51 & $\ldots$ & & & $13.079(013)$ & \\
\hline 8094.51 & $13.386(026)$ & $13.443(017)$ & 13.057(017) & $13.202(013)$ & $13.478(016)$ \\
\hline 8095.49 & $13.500(026)$ & $13.502(017)$ & $13.092(017)$ & $13.206(013)$ & $13.460(016)$ \\
\hline 8097.51 & $\ldots$ & $13.774(017)$ & $13.236(017)$ & $13.328(013)$ & $13.550(015)$ \\
\hline 8098.52 & $\ldots$ & $13.885(017)$ & $13.264(017)$ & 13.341(013) & $13.531(015)$ \\
\hline 8104.48 & $14.712(026)$ & $14.522(017)$ & 13.614(017) & 13.464(013) & $13.428(015)$ \\
\hline 8104.50 & $\ldots$ & $14.520(017)$ & 13.618(017) & $13.468(013)$ & $13.424(015)$ \\
\hline 8124.47 & $\ldots$ & $15.753(022)$ & $14.608(018)$ & $14.252(021)$ & $14.012(018)$ \\
\hline 8124.47 & $\cdots$ & $\ldots$ & $\ldots$ & $14.271(018)$ & $\ldots$ \\
\hline 8253.83 & $\cdots$ & 17.492(019) & $17.539(018)$ & 18.145(019) & $18.053(045)$ \\
\hline 8272.82 & $\cdots$ & $17.958(018)$ & $17.796(018)$ & 18.317(020) & $18.227(041)$ \\
\hline 8292.76 & $\cdots$ & $18.049(043)$ & $17.899(044)$ & $18.455(086)$ & $18.787(218)$ \\
\hline 8299.87 & $19.588(059)$ & $18.118(018)$ & $18.242(018)$ & $18.765(017)$ & $18.682(034)$ \\
\hline 8299.87 & $\ldots$ & & & $18.750(027)$ & $18.610(040)$ \\
\hline 8307.80 & $\cdots$ & $18.299(023)$ & $18.286(023)$ & $18.989(047)$ & $18.824(046)$ \\
\hline 8307.80 & $\cdots$ & $\ldots$ & $18.394(025)$ & $18.916(021)$ & $18.782(043)$ \\
\hline 8322.83 & $\cdots$ & $18.554(027)$ & $18.587(029)$ & $19.160(047)$ & $18.961(050)$ \\
\hline 8334.76 & $\cdots$ & $\ldots$ & $18.756(027)$ & $\ldots$ & $19.185(088)$ \\
\hline 8335.74 & $\cdots$ & 18.842(021) & 18.767(023) & 19.531(038) & $18.947(061)$ \\
\hline 8347.70 & $\cdots$ & $18.771(076)^{\mathrm{a}}$ & $18.941(048)^{\mathrm{a}}$ & $19.550(057)^{\mathrm{a}}$ & $19.378(131)^{\mathrm{a}}$ \\
\hline 8347.74 & $\ldots$ & $18.829(092)^{\mathrm{a}}$ & $18.900(046)^{\mathrm{a}}$ & $19.635(073)^{\mathrm{a}}$ & $19.318(125)^{\mathrm{a}}$ \\
\hline 8353.69 & $\cdots$ & 18.932(028) & 18.981(026) & 19.764(041) & $19.291(066)$ \\
\hline 8355.69 & $\cdots$ & 18.864(018) & 19.053(019) & 19.711(018) & $19.235(025)$ \\
\hline 8414.61 & $\ldots$ & $19.960(035)^{\mathrm{a}}$ & $20.114(038)^{\mathrm{a}}$ & $20.853(042)^{\mathrm{a}}$ & $20.107(059)^{\mathrm{a}}$ \\
\hline 8414.61 & $\cdots$ & 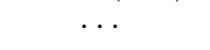 & & 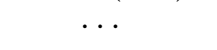 & $20.048(070)^{\mathrm{a}}$ \\
\hline 8420.54 & $\cdots$ & 19.888(044) & 19.931(051) & $20.527(121)$ & $\ldots$ \\
\hline 8459.51 & $\cdots$ & $20.541(149)^{\mathrm{a}}$ & $20.904(231)^{\mathrm{a}}$ & & $\cdots$ \\
\hline 8687.82 & $\cdots$ & & $23.198(114)^{\mathrm{a}}$ & & \\
\hline
\end{tabular}

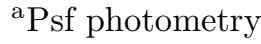


TABLE 6. SN 1991T photometry.

\begin{tabular}{|c|c|c|c|c|c|}
\hline $\begin{array}{c}\text { J.D. - } \\
2440000\end{array}$ & $\begin{array}{c}U \\
\operatorname{mag}(\text { m.e. })\end{array}$ & $\begin{array}{c}B \\
\operatorname{mag}(\text { m.e. })\end{array}$ & $\begin{array}{c}V \\
\operatorname{mag}(\text { m.e. })\end{array}$ & $\begin{array}{c}R \\
\operatorname{mag}(\text { m.e. })\end{array}$ & $\begin{array}{c}I \\
\operatorname{mag}(\text { m.e. })\end{array}$ \\
\hline 8363.77 & $\cdots$ & $12.712(016)$ & $12.618(016)$ & $12.526(015)$ & \\
\hline 8364.76 & $\cdots$ & $12.449(016)$ & $12.362(016)$ & $12.299(015)$ & \\
\hline 8365.66 & $11.610(026)$ & $12.362(016)$ & $12.256(016)$ & $12.134(015)$ & $12.215(017)$ \\
\hline 8365.65 & $\ldots$ & $\ldots$ & $\ldots$ & $12.159(015)$ & \\
\hline 8366.67 & $\ldots$ & $12.152(016)$ & $12.061(016)$ & $11.971(015)$ & \\
\hline 8372.70 & $\ldots$ & $11.748(016)^{\mathrm{b}}$ & $\ldots$ & $11.533(015)$ & $11.693(017)$ \\
\hline 8374.64 & $11.282(026)^{\mathrm{a}}$ & $11.719(016)^{b}$ & $11.573(016)^{\mathrm{b}}$ & $11.511(015)$ & $11.690(017)$ \\
\hline 8374.64 & $11.304(026)^{\mathrm{a}}$ & $11.707(016)^{b}$ & $11.560(016)^{\mathrm{b}}$ & $11.519(015)$ & $11.694(017)$ \\
\hline 8375.65 & $11.370(026)^{\mathrm{a}}$ & $11.715(016)^{b}$ & $11.568(016)^{\mathrm{b}}$ & $11.529(015)$ & $11.689(017)$ \\
\hline 8375.65 & $11.270(026)^{\mathrm{a}}$ & $11.720(016)^{b}$ & $11.537(016)^{\mathrm{b}}$ & $11.509(015)$ & $11.676(017)$ \\
\hline 8376.69 & $11.279(026)^{\mathrm{a}}$ & $11.696(016)^{b}$ & $11.542(016)^{\mathrm{b}}$ & $11.474(015)$ & \\
\hline 8376.69 & $11.233(026)^{\mathrm{a}}$ & $11.741(016)^{b}$ & $11.527(016)^{\mathrm{b}}$ & $11.475(015)$ & \\
\hline 8376.69 & $\ldots$ & & & & $11.690(017)$ \\
\hline 8377.62 & $11.329(026)^{\mathrm{a}}$ & $11.694(016)^{b}$ & $11.511(016)^{\mathrm{b}}$ & $11.435(015)$ & $11.664(017)$ \\
\hline 8377.62 & $\ldots$ & $11.711(016)^{b}$ & $11.482(016)^{\mathrm{b}}$ & $11.435(015)$ & $11.665(017)$ \\
\hline 8380.70 & $\cdots$ & 11.832(016) & $11.525(016)$ & $11.466(015)$ & $11.788(017)$ \\
\hline 8380.69 & $\ldots$ & $\ldots$ & $11.529(016)$ & $11.479(015)$ & $11.805(018)$ \\
\hline 8381.60 & $\ldots$ & $11.836(016)$ & $11.535(016)$ & $11.518(015)$ & $11.847(017)$ \\
\hline 8381.60 & $\ldots$ & $11.857(016)$ & $\ldots$ & $11.501(015)$ & $11.846(017)$ \\
\hline 8382.67 & $\ldots$ & 11.912(016) & $11.577(016)$ & $11.565(015)$ & $11.915(017)$ \\
\hline 8382.68 & $\ldots$ & $11.919(016)$ & $11.575(016)$ & $11.552(015)$ & $11.917(017)$ \\
\hline 8383.58 & $11.652(026)$ & $11.959(016)$ & $11.605(016)$ & $11.589(015)$ & $11.966(017)$ \\
\hline 8383.58 & & $11.971(016)$ & $11.583(016)$ & $11.585(015)$ & $11.967(017)$ \\
\hline 8385.63 & $\ldots$ & $12.137(016)$ & $11.643(016)$ & $11.730(015)$ & $12.051(017)$ \\
\hline 8385.62 & $\ldots$ & & $\ldots$ & $11.729(015)$ & $12.049(017)$ \\
\hline 8386.55 & $\cdots$ & $12.248(016)$ & $11.764(016)$ & $11.811(015)$ & $12.087(017)$ \\
\hline 8386.55 & $\ldots$ & $12.208(016)$ & $\ldots$ & & 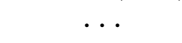 \\
\hline 8388.57 & $12.381(026)$ & $12.408(016)$ & $11.873(016)$ & $11.917(015)$ & \\
\hline 8390.50 & $12.750(026)$ & $12.603(016)$ & $11.974(016)$ & $11.982(015)$ & $12.076(017)$ \\
\hline 8390.50 & $12.778(026)$ & $12.600(016)$ & $11.971(016)$ & $11.976(015)$ & $12.062(017)$ \\
\hline 8393.62 & $13.222(026)$ & $12.965(016)$ & $12.150(016)$ & $12.029(015)$ & $12.022(017)$ \\
\hline 8395.55 & $\ldots$ & 13.131(016) & $12.223(016)$ & $\ldots$ & 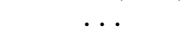 \\
\hline 8395.56 & $\ldots$ & $13.149(016)$ & $12.247(016)$ & $\cdots$ & $\cdots$ \\
\hline 8405.59 & $\ldots$ & $14.071(016)$ & $12.731(016)$ & $12.378(015)$ & $12.098(017)$ \\
\hline 8405.59 & $\cdots$ & $14.071(017)$ & $12.735(016)$ & $12.379(015)$ & $12.111(017)$ \\
\hline 8414.62 & $14.610(026)$ & $14.302(016)$ & $13.123(016)$ & $12.820(015)$ & $12.421(017)$ \\
\hline 8414.62 & & $14.307(016)$ & & & \\
\hline 8420.47 & $14.862(026)$ & $14.556(016)$ & $13.423(016)$ & $13.102(015)$ & $12.819(017)$ \\
\hline 8431.49 & $15.032(030)$ & $14.770(017)$ & $13.744(016)$ & $13.527(015)$ & $13.386(017)$ \\
\hline 8431.49 & $15.070(030)$ & $14.746(017)$ & $13.754(016)$ & & \\
\hline 8438.50 & $\ldots$ & $14.863(016)$ & 13.936(016) & $\ldots$ & $13.712(017)$ \\
\hline 8439.47 & $\cdots$ & 14.931(016) & $13.986(016)$ & $13.830(015)$ & $13.738(017)$ \\
\hline 8439.47 & $\cdots$ & $14.919(016)$ & $14.018(016)$ & $13.824(015)$ & $13.749(017)$ \\
\hline 8440.46 & $\cdots$ & $14.897(016)$ & $14.059(016)$ & $13.859(001)$ & $13.821(017)$ \\
\hline 8440.46 & $\cdots$ & $14.917(016)$ & $14.058(016)$ & $13.872(015)$ & $13.790(017)$ \\
\hline 8442.48 & $15.265(026)$ & $14.888(016)$ & $14.045(016)$ & $13.888(015)$ & $13.778(017)$ \\
\hline 8445.46 & $\ldots$ & $\ldots$ & $14.139(016)$ & $\ldots$ & $\ldots$ \\
\hline 8445.47 & $\cdots$ & $14.934(017)$ & $14.143(016)$ & $\cdots$ & $\cdots$ \\
\hline
\end{tabular}


TABLE 6. (continued)

\begin{tabular}{cccccc}
\hline $\begin{array}{c}\text { J.D. }- \\
2440000\end{array}$ & $\begin{array}{c}U \\
\operatorname{mag}(\text { m.e. })\end{array}$ & $\begin{array}{c}B \\
\operatorname{mag}(\text { m.e. })\end{array}$ & $\begin{array}{c}V \\
\operatorname{mag}(\text { m.e. })\end{array}$ & $\begin{array}{c}R \\
\operatorname{mag}(\mathrm{m.e.})\end{array}$ & $\begin{array}{c}I \\
\operatorname{mag}(\mathrm{m} . \mathrm{e} .)\end{array}$ \\
\hline 8459.49 & $\ldots$ & $15.115(016)$ & $14.476(016)$ & $14.407(015)$ & $14.538(017)$ \\
8628.83 & $\ldots$ & $17.496(019)$ & $17.484(018)$ & $\ldots$ & $17.939(040)$ \\
8629.83 & $\ldots$ & $17.513(018)$ & $17.475(018)$ & $\ldots$ & $17.951(046)$ \\
8677.78 & $\ldots$ & $\ldots$ & $18.116(022)$ & $\ldots$ & $18.367(045)$ \\
8687.85 & $\ldots$ & $\ldots$ & $18.343(017)$ & $19.086(022)$ & $18.489(026)$ \\
8691.78 & $\ldots$ & $18.445(018)^{\mathrm{a}}$ & $18.357(019)^{\mathrm{a}}$ & $19.080(025)^{\mathrm{a}}$ & $18.489(031)^{\mathrm{a}}$ \\
8720.77 & $\ldots$ & $18.834(023)^{\mathrm{a}}$ & $18.765(022)^{\mathrm{a}}$ & $\ldots$ & $18.952(034)^{\mathrm{a}}$ \\
8731.63 & $\ldots$ & $18.916(078)^{\mathrm{a}}$ & $18.947(047)^{\mathrm{a}}$ & $\ldots$ & $\ldots$ \\
8731.62 & $\ldots$ & $\ldots$ & $19.010(052)^{\mathrm{a}}$ & $\ldots$ & $\ldots$ \\
8776.53 & $20.346(250)^{\mathrm{a}}$ & $19.654(078)^{\mathrm{a}}$ & $19.662(057)^{\mathrm{a}}$ & $20.271(102)^{\mathrm{a}}$ & $19.374(119)^{\mathrm{a}}$ \\
8776.54 & $\ldots$ & $19.773(097)^{\mathrm{a}}$ & $19.684(063)^{\mathrm{a}}$ & $20.162(097)^{\mathrm{a}}$ & $19.359(113)^{\mathrm{a}}$ \\
\hline \hline
\end{tabular}

${ }^{\text {a }}$ Psf photometry

${ }^{\mathrm{b}}$ Photometry calibrated using the core of NGC 4527 
TABLE 7. Peak photometric magntiudes.

\begin{tabular}{|c|c|c|}
\hline Filter & Magnitude & JD - 2440000 \\
\hline \multicolumn{3}{|c|}{ SN 1990N } \\
\hline$U$ & $12.38 \pm 0.02$ & $8081.8 \pm 1.0$ \\
\hline$B$ & $12.76 \pm 0.03$ & $8082.7 \pm 0.5$ \\
\hline$V$ & $12.70 \pm 0.02$ & $8084.2 \pm 0.5$ \\
\hline$R$ & $12.69 \pm 0.02$ & $8082.9 \pm 0.5$ \\
\hline$I$ & $12.94 \pm 0.02$ & $8080.7 \pm 1.0$ \\
\hline \multicolumn{3}{|c|}{ SN 1991T } \\
\hline$U$ & $11.26 \pm 0.02$ & $8374.0 \pm 1.0$ \\
\hline$B$ & $11.70 \pm 0.02$ & $8375.7 \pm 0.5$ \\
\hline$V$ & $11.51 \pm 0.02$ & $8378.3 \pm 0.5$ \\
\hline$R$ & $11.46 \pm 0.02$ & $8377.1 \pm 0.5$ \\
\hline$I$ & $11.67 \pm 0.02$ & $8375.3 \pm 1.0$ \\
\hline
\end{tabular}


TABle 8. Pskovskii and Phillips parameters.

\begin{tabular}{cclccc}
\hline \hline Object & $\begin{array}{c}\Delta m_{15}(B) \\
(\mathrm{mags})\end{array}$ & $\begin{array}{c}\beta \\
(\mathrm{mags} / \text { 100days })\end{array}$ & $\begin{array}{c}\text { Light curve range } \\
\text { (days) }\end{array}$ & $\begin{array}{c}\gamma \\
\text { (mags/100days) }\end{array}$ & $\begin{array}{c}\text { Light curve range } \\
\text { (days) }\end{array}$ \\
\hline SN 1990N & $1.03 \pm 0.06$ & $11.03 \pm 0.13$ & $11-22$ & $1.380 \pm 0.004$ & $42-273$ \\
SN 1991T & $0.95 \pm 0.05$ & $10.21 \pm 0.09$ & $10-20$ & $1.381 \pm 0.007$ & $55-254$ \\
Leibundgut & 1.10 & 11.29 & $\cdots$ & 1.676 & $\cdots$ \\
\hline \hline
\end{tabular}

